\title{
Light Emitting Transistors Using Silicon Quantum Dots in an Organic Matrix
}

\author{
A THESIS \\ SUBMITTED TO THE FACULTY OF THE GRADUATE SCHOOL \\ OF THE UNIVERSITY OF MINNESOTA \\ BY
}

Gagan Aggarwal

IN PARTIAL FULFILLMENT OF THE REQUIREMENTS

FOR THE DEGREE OF

MASTER SCIENCE

Stephen. A. Campbell

January 2011 
(C) Gagan Aggarwal 2011 


\section{Acknowledgement}

I dedicate this thesis to my parents. This degree would not have been attainable without their unconditional love and support that motivated me to come to the United States to pursue my dreams. I owe everything to them.

A very special thanks to my advisor Professor Stephen A. Campbell, whose guidance, support and encouragement during this course of work is invaluable. Thank you for giving me the chance to learn and work under your guidance to obtain this Masters Degree and always guiding me through the tough times of my life.

I would also like to express thanks to my committee members Professor Beth Stadler and Professor Ted Higman for reviewing my dissertation and providing me invaluable support and feedback.

I would like to express special thanks to my wife Shikha who has always been a source of support and encouragement. This would not have been possible without her. Love you honey!

I would also like to thank my group members Maryam Jalali, Rick Liptak, Sangho Song, Min Woo Jang, Brijesh Kumar, Kush Nagaich and Dan Yu. Their assistance, helpful discussion and friendship through the years were helpful.

Special thanks to my friends Anand and Shweta for providing their valuable suggestions and inputs time to time.

Last but not the least, thanks to all those visible and invisible hands that made this day possible. 


\begin{abstract}
As the demands on our world's energy resources continue to grow, alternative high efficiency materials such as quantum confined silicon nano particles are desirable for their potential low cost application in general white light illumination. In optical displays, and in on-chip optical interconnects. Many fabrication and passivation techniques have been developed that produce silicon nano particles with high photogenerated quantum yield. However, high electrically generated silicon nano particles quantum efficiency has eluded our society. Predominantly due to lack of surface passivation and device fabrication techniques that preserves the nano particle properties. Hence, in this work we have developed a Hybrid MESFET where we can control the amount of light from the device by controlling the gate voltage and also with the use of the organics we can also preserve the electroluminescent properties of the silicon nano particles.

The passivation of nonthermal plasma fabricated silicon nano particles with surface oxide grown was done under UV exposure. The surface oxidized silicon nano particles $\left(\mathrm{Si} / \mathrm{SiO}_{2}\right)$ surface chemistry was composed predominately of $\mathrm{SiO}_{2}$.

Hybrid MESFET's were fabricated with the use of couple of organic materials (PVK and PEDOT:PSS) and in organics (ITO and Aluminum). Choice of materials were based upon the previous work done by another students in the same group and also based upon the band alignment of the different layers and also upon the process ability of those materials.
\end{abstract}


The devices once fabricated were tested for the functioning using parameter analyzer and the light output was recorded with silicon photodiode. Also for the verification of the results simulation was done using PADRE simulator. 


\section{Table of Contents}

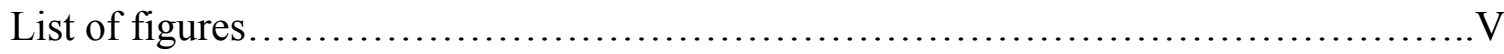

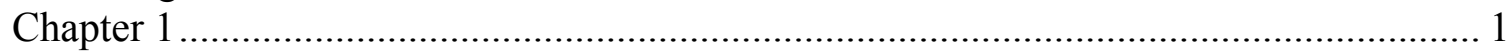

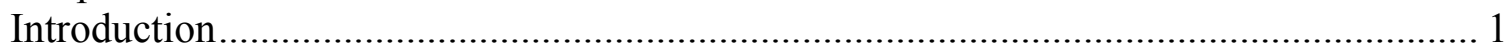

1.1 Luminescent Silicon Nanoparticles .....................................................................

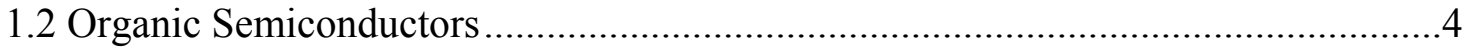

1.3 Hybrid Organic Light Emitting Diode .................................................................

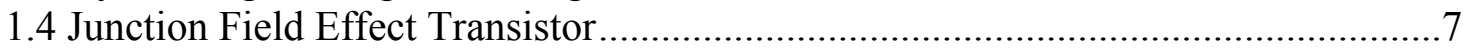

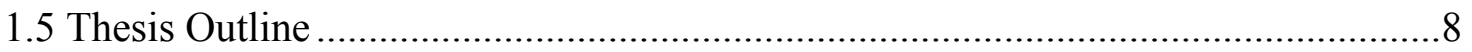

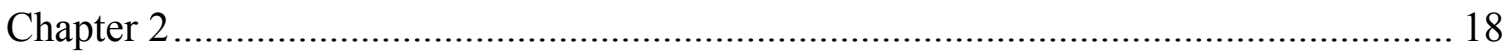

Production and Synthesis of Nano particles ……………........................................... 18

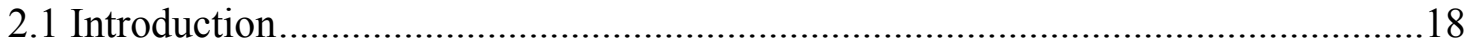

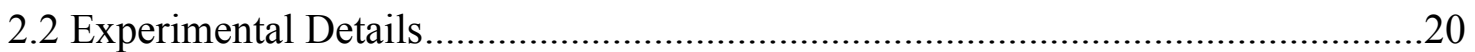

2.2.1 Silicon Nano Particles Non Thermal Plasma Fabrication and Passivation...... 20

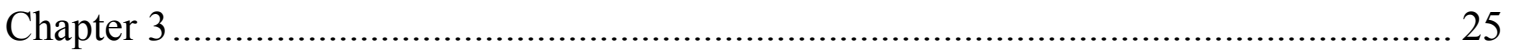

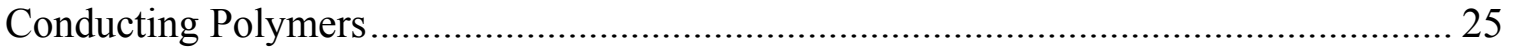

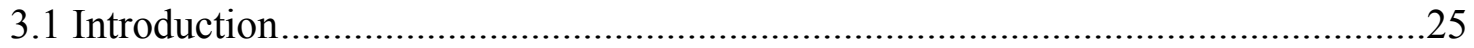

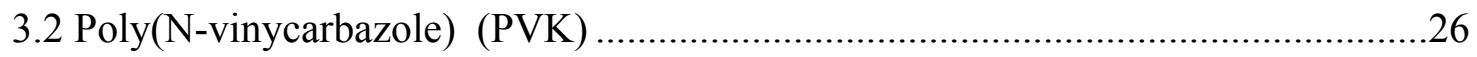

3.3 Poly(3,4-ethylenedioxythiophene):poly(styrenesulfonate) (PEDOT:PSS) .............27

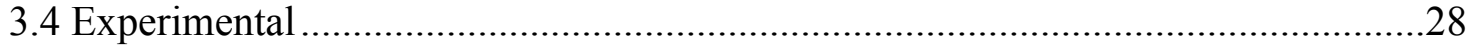

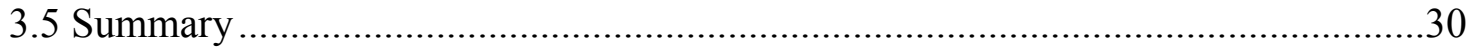

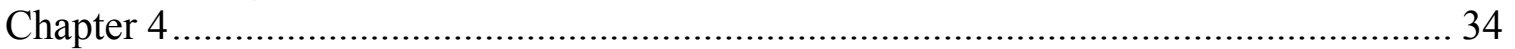

Hybrid Light Emitting Transistors............................................................................... 34

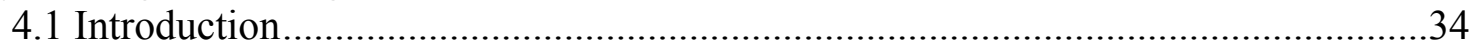

4.2 Metal Semiconductor Field Effect Transistor (MESFET) ......................................34

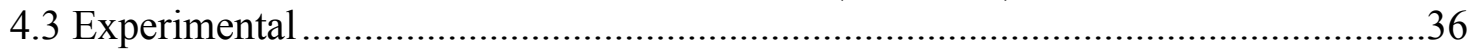

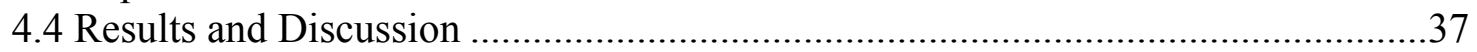

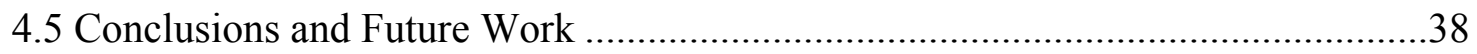

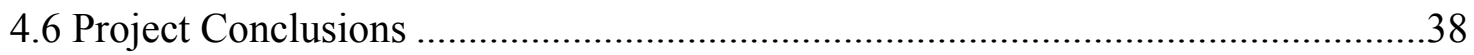

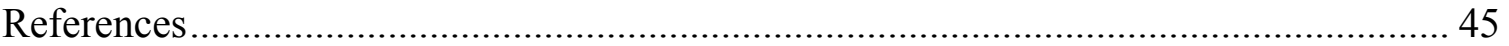




\section{List of Figures}

Figure 1.1 [36] Spectrum of Light Emission from nano particles under UV excitation . 10 Figure 1.2 [24] Plasma system to produce nano particles ............................................ 11

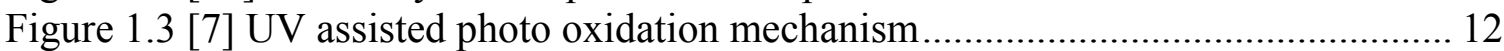

Figure 1.4: Commonly used semiconducting polymers ................................................. 13

Figure 1.5: Structure of poly(3-hexylthiophene) (P3HT) ……................................... 13

Figure 1.6: Structure of poly(3-hexylthiophene) (P3HT) ............................................... 14

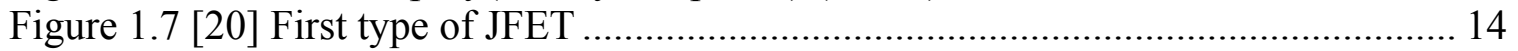

Figure 1.8 [20] Cross section of a $\mathrm{n}$ channel p-n junction FET ..................................... 15

Figure 1.9: Single layer hybrid device (a) band diagram showing respective workfunction, conduction, valance, HOMO and LUMO energies for the different

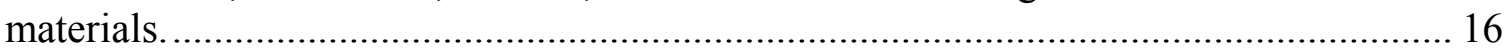

Figure 1.10: Multilayer hybrid device (a) band diagram showing the respective workfunction conduction, valance, HOMO, LUMO energies for the different materials, and (b) layout under forward bias. Within this figure EL denotes emissive layer. .......... 17

Figure 2.1: Description of Chemical Reaction on Silicon Nano Particles........................ 23

Figure 2.2: Photoluminescence Spectrum of Dodecene Coated Silicon Quantum Dots. . 24

Figure 3.1: Several conjugated polymer repeat unit .................................................... 31

Figure 3.2: $\mathrm{N}$-vinalcarbazole and Poly(N-vinycarbazole $)$............................................ 32

Figure 3.3: Poly(3,4-ethylenedioxythiophene):poly(styrenesulfonate) (PEDOT:PSS).... 32

Figure 3.4: Photoresist Pattern Peeled off as PEDOT:PSS film is washed away ............. 33

Figure 3.5: Patterned Gate over PEDOT:PSS.............................................................. 33

Figure 4.1 [88]: Cross section of a N channel MEsfet with semi-insulating substrate..... 40 Figure 4.2 [88]: Idealized Energy band Diagram of the substrate- channel- metal in the n-

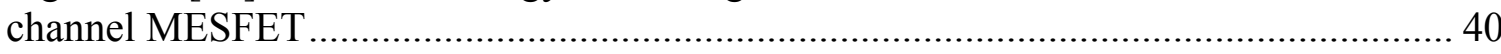

Figure 4.3 [88]: Channel space charge region of an enhancement mode MESFET for (a)

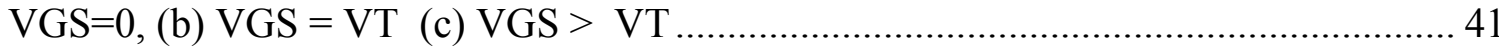

Figure 4.4: PEDOT:PSS is spin coated over Aluminum Gates ....................................... 42

Figure 4.5: Device after spin coating nano particles and depositing top aluminum

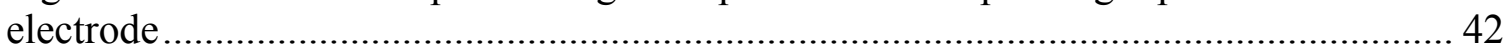

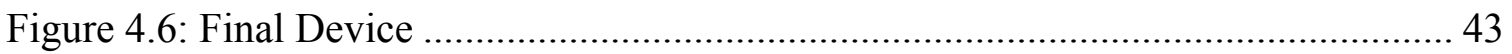

Figure 4.7: Electrical Characteristic of the devices ..................................................... 43

Figure 4.8: Optical characteristics of the device............................................................ 44

Figure 4.9: Simulations results by using PADRE simulator............................................. 44 


\section{Chapter 1}

\section{Introduction}

This chapter provides review of relevant topics to the research in this thesis. Luminescent nano particles are introduced first followed by Organic Semiconductors and Field Effect Transistors. In the early $20^{\text {th }}$ century light emission from a solid state material powered by a voltage source was observed. This "light emission" occurred at room temperature and was fundamentally different than incandescence (which is visible EM radiation emitted by a material once it is heated to high temperatures). This room temperature luminescence which would later be called electroluminescence was first observed in silicon carbide devices.

\subsection{Luminescent Silicon Nanoparticles}

The possibility of using silicon nanoparticles as an efficient light source is attractive for potential applications in optoelectronics, optical interconnections, and electroluminescence devices because of their compatibility with existing silicon processing/manufacturing infrastructure technology for ultra large-scale integrated circuits. In nanotechnology, a particle is defined as a small object that behaves as a whole unit in terms of its transport and properties. Nanoparticles are sized between 1 and 100 nanometers, though the size limitation can be restricted to two dimensions. Visible photoluminescence as shown in figure 1.1 has been observed from silicon nanoparticles due to quantum-size confinement, although, in principle it is not possible to obtain light emission from bulk silicon due to its indirect band transition [1], [2], [3], [4] and [5] 
Luminescent silicon nanoparticles are crystalline inorganic semiconductor materials that, unlike bulk crystalline silicon, emit light by spatially confining electron-hole pairs (also called excitons) to the nanoparticles. The quantum confinement effect is produced by the discretization of the quasi continuum of the band states that describes the bulk semiconductor that occurs when the material size approaches that of the Bohr radius, which for silicon is around $2.5 \mathrm{~nm}$. The discrete energy levels inherently increase the exciton binding energy, which at room temperature is a few $\mathrm{meV}$, to a few $\mathrm{eV}$. Since silicon is an indirect material the exciton remains trapped within the nanoparticle until a lattice vibration or phonon of appropriate energy collides with the exciton and through conservation of momentum, the exciton recombines releasing light with energy that depends on the size of the nanoparticle, but is always higher than the bulk silicon band gap. Because this process is slow, radiative lifetimes in silicon nanoparticles is long, typically of order milliseconds. Thus, the lifetime for nonradiative recombination pathways must be at least this long for emission to be observed.

Electroluminescence from silicon nanocrystals was first observed by the thermal annealing and thermal oxidation of ultrathin hydrogenated amorphous silicon films deposited using various deposition techniques [6]. Such in situ techniques are advantageous as they have improved quantum efficiency of silicon nanoparticles by passivating dangling bonds at the interface between the silicon nanoparticles and the host matrix, thereby reducing the number of non-radiative pathways [7] and [8]. These processes for producing nanoparticles also reduce the particles agglomeration and helps in reducing the detrimental pin holes and the non-uniform carrier flux within the device. 
However, these processes have several drawbacks. First, they have limited control over the size and the shape of the nano particles which results in a very unstable emission [4], [9], [10], [11] and [12]. Second, manufacturing process generally requires high temperature anneals, making it unsuitable for use on the top of existing design using soft temperature sensitive materials. Finally, the range of materials in which such nanoparticles can be made is highly limited and so one cannot freely select the materials in the device. The ideal silicon nanoparticles would be uniformly sized and shaped with strong quantum confined optical properties enclosed within a high carrier transport material(s) that optimizes the carrier transport to the nanoparticles.

Uniformly sized and shaped free standing luminescent silicon nanoparticles are readily fabricated at high rates using a scalable single-step in-flight low-pressure non-thermal plasma process as shown in figure 1.2 [36]. The silicon nano particles are created in the plasma through electron impact disassociation of silane gas, leading to cluster formation and nanocrystal growth. The size is tuned from 5 to $8 \mathrm{~nm}$ by adjusting the residence time or silane partial pressure in the system. The plasma system is designed in such a way that particles must flow through a region of high plasma density where they are annealed to remove crystal imperfections. The silicon nanoparticles are collected downstream of the plasma by either implantation on the device substrate or they are collected on the stainless steel mesh and are post processed by various techniques. Si has several major advantages compared to III-V and II-VI quantum dot approaches due to its ready availability, low cost, low toxicity for consumer products, and compatibility with conventional integrated circuit processing. 
The as-produced silicon nanoparticles commonly have poor optical properties due to incomplete hydrogen surface passivation leaving non-radiative recombination centers. Hence, the freestanding silicon nanoparticles surface must be passivated to optimize the silicon nanoparticles optical properties prior to device fabrication. Figure 1.3 shows the UV assisted photo oxidation process used to passivate the nano particles

\subsection{Organic Semiconductors}

Organic semiconducting materials have been synthesized and studied for over many decades [13]. In the 1950s, drift mobility measurements and the photoconductivity (PC) response of small molecules such as anthracene were examined [13] and [14] and although these materials showed semiconducting properties (i.e., conductivities in the range of $10^{-9}-10^{-6} \mathrm{~S} \mathrm{~cm}^{-1}$ ) [15], their performance and stability were poor. However, with improvements in synthesis and processing techniques of new classes of molecules such as conjugated polythiophenes in the past two decades [16], the prospects of commercially using organic semiconductors (OSCs) in applications such as organic lightemitting diodes (OLEDs), field-effect transistors (OFETs) and the solar cells is now greater than ever.

Conjugated material is the most important class of organic semiconductors [17] and can be categorized into two main groups [15] of conjugated polymers and conjugated oligomers. Polymers, such as polythiophenes consist of many identical units bonded together in a long chain, and are macromolecular in nature, whereas oligomers like oligothiophenes consist of only a few units. In these materials, carbon $p_{z}$ orbitals overlap and the $\pi$ electrons become delocalized on the molecule, forming the so-called $\pi$ - 
conjugated system [18]. The alternating single and double bonds in polymer drawings represent these $\pi$ electrons. There are other classes of $\pi$-conjugated OSCs, such as pentacene and fullerenes which have demonstrated good semiconducting properties. Figure 1.4 shows some important organic semiconductors. An important conjugated polymer, which has been our material of choice for all the experiments and analysis presented in this thesis, is regioregular poly(3-hexylthiophene) (P3HT). Figure 1.5 shows a sketch of this molecule. We have also used another polymer Poly(9-vinylcarbazole) (PVK) shown in Figure 1.6 which is a $\pi$-conjugated polymer. Nanoparticles are mixed with PVK and are spin coated to get smooth films. It is it is now well known that PVK is an effective host of hybrid silicon quantum dot LEDs [6]. The polymer around the nanoparticles not only helps in getting a uniform film but also serves the purpose of charge transport.

\subsection{Hybrid Organic Light Emitting Diode}

Pi-conjugated polymers and small molecules are commonly found in OLEDs have intensely been pursued as potential conductive hosts for improving luminescent $\mathrm{np}$ quantum efficiency. Similar to nano particles OLED's rely on the simultaneous injection and recombination of electron and holes to generate Electroluminescence. Hence OLED's support high carrier transport such that nano particles located within an LOED will, in theory, be showered with a high flux of carriers and therefore have a high probability of generating electroluminescence.

Electroluminescence from CdSe nano particles have been reported by many different authors who have incorporated luminescent $\mathrm{CdSe}$ nanoparticles in to various single and 
multilayer OLED architectures [19], [20], [21], [22] and [23]. Hybrid device architecture and corresponding materials are predominantly chosen to optimize energy band alignments thereby enhancing the desired emission, while material thermal stability and processibility are also considered.

Single layer devices composed of nanoparticles dispersed in organics are commonly fabricated using composite organic - nanoparticles solution spin coating on indium tin oxide (ITO) and subsequent thermal evaporation and patterning of the top cathode contact [19], [24], and [25]. However since the nanoparticles are located within the organic material, it must support both electron and the hole transport to the nano particles. For this to occur efficiently, the organics and the nano particles must form a type- I hetrojunction as shown in the figure 1.9 where the highest occupied molecular orbital (HOMO) and lowest occupied molecular orbital (LUMO) of the organic straddle the conduction band $(\mathrm{Ec})$ and the valance band $(\mathrm{Ev})$ of the nano particle. Based on the type-I energy band alignment the nano particles serves as radiative recombination center such that lower energy nano particle emission occurs preferentially over the higher energy organic emission [19], [25] and [26].

Since very few if any organic material possess the ability to efficiently transport both holes and the electrons, multilayer devices composed of nano particles embedded between electron and hole transport layers have also been pursued. Such multilayer devices are fabricated by spin coating thin films of nano particles over a hole transporting layer (HTL) initially coated on ITO or using preferential phase segregation of nanoparticles from a hole transporting small molecules during spin coating [22], [27], and 
[28]. The electron transport layer [ETL] and cathode are then deposited by thermal evaporation to complete the device. The organics used in a multilayer hybrid device are chosen to form a type II hetero junction with the nano particles as shown in figure 1.10 where the organics HOMO-LUMO gap staggers the nano particles Ec-Ev gap. The type II band alignment favors carrier injection in to the nano particles while simultaneously blocking the carrier transport out of the nano particles. Hence, carriers are trapped within the nano particles thereby favoring exciton recombination within the nano particle layer. Furthermore, the dedicated ETL and HTL balance charge injection to the nano particle thereby minimizing exciton loss to non-radiative auger processes [29], [30] and [31]. In this project I have used PEDOT:PSS is used as a hole conducting layer. PEDOT:PSS is a water based polymer that is spin coated on ITO. More about this polymer and also its coating process is discussed in later chapters.

\subsection{Junction Field Effect Transistor}

The concept of the field effect phenomenon was the basis for the first proposed solid state transistor. The phenomenon of modulating the conductance of a semiconductor by an electric field applied perpendicular to the surface of a semiconductor is called the field effect. Field effect transistors predate the bipolar transistor by approximately 20 years. The first modern day field effect device, the Junction Field Effect Transistor (J-FET), was proposed and analyzed by Shockley in 1952. Operational J-FETs were subsequently built by Dacey and Ross in 1953 [32].

The J-FET was initially named the unipolar transistor to distinguish it from the bipolar junction transistor and to emphasize that only one type of carrier is involved in the 
operation. J-FETs, in conjunction with other circuit elements, is capable of voltage and signal power gain. The current in a JFET is through a semiconducting region known as the channel, with ohmic contacts at each end. The basic transistor action is the modulation of the channel conductance by electric field that is perpendicular to the channel. The modulating electric field is induced by the space charge region of the reverse biased pn junction or in the closely related MESFET, a reverse biased Schottky barrier junction, and is therefore a function of function of gate voltage. The modulation of the channel conductance by the gate voltage modulates the channel current.

JFETs are widely used as active components in the front-end electronics of silicon radiation detectors due to their low-noise figures and high radiation hardness [33] and [34]. JFETs are of two types $\mathrm{p}$ channel and $\mathrm{n}$ channel transistor based on the doping of the channel. In this work I have fabricated the $\mathrm{p}$ channel MESFET. The channel is formed by the $\mathrm{p}$ type polymer and the gate is formed by patterning aluminum. Poly(9vinyl carbazole) (PVK) was then added to the nanoparticles. PVK is an effective host of hybrid silicon quantum dot LED's [35].

\subsection{Thesis Outline}

The work contained in this thesis will focus on fabricating Light Emitting Junction Field Effect Transistor (JFELET). These are hybrid transistor fabricated using both organic and inorganic materials. Chapter 2 discusses the silicon nanoparticles nonthermal plasma fabrication and passivation process. The nonthermal plasma techniques mass produces the highly crystalline silicon nano particles with precise size control and the passivation process is done to attach the long carbon chain on the nano particles. Chapter 3 presents 
the insight to the various organic materials that can be sued for fabricated the semiconducting devices. These organics materials are cheap and can be used to fabricate the flexible devices. Chapter 4 presents the experimental investigations, results and summarizes this thesis work and provides suggestions and discusses the future work. 
Figure 1.1 [36] Spectrum of Light Emission from nano particles under UV excitation

\section{Under an excitation at $365 \mathrm{~nm}$}

$\mathrm{PL}$ emission peaks are at $652 \mathrm{~nm}, \quad \mathrm{~nm}, 5 / 2 \mathrm{~nm}, 441 \mathrm{~nm}$, respectively.

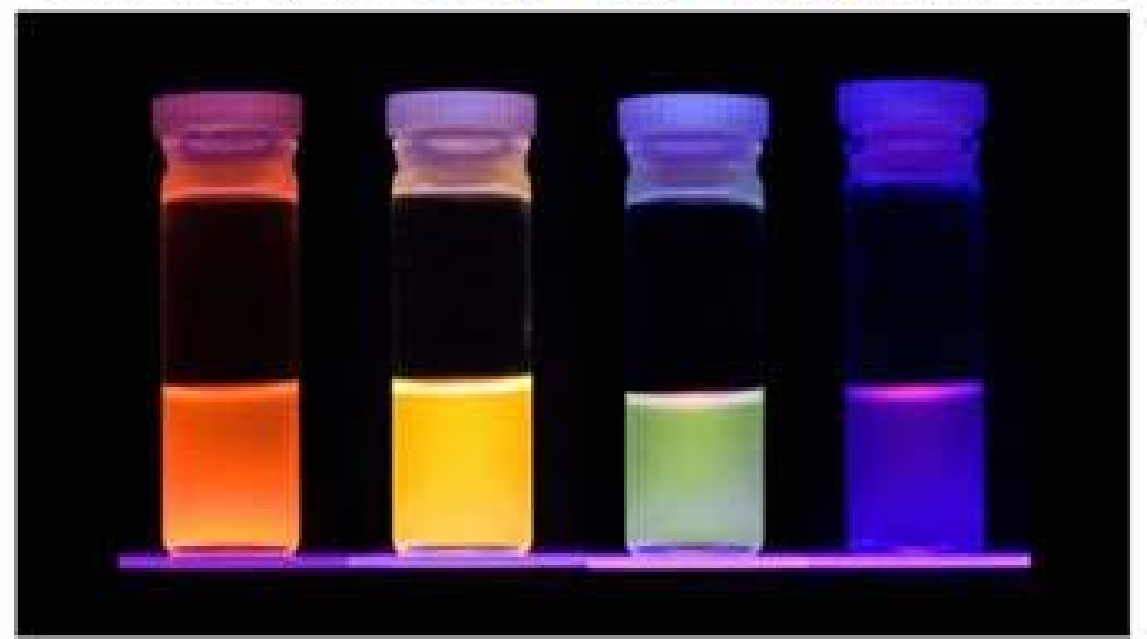

Samples are radiated from underneath by a UV lamp, of which the emission is centered at $365 \mathrm{~nm}$. A $385 \mathrm{~nm}$ short-pass fister is placed between the UV lamp and samples. 
Figure 1.2 [24] Plasma system to produce nano particles

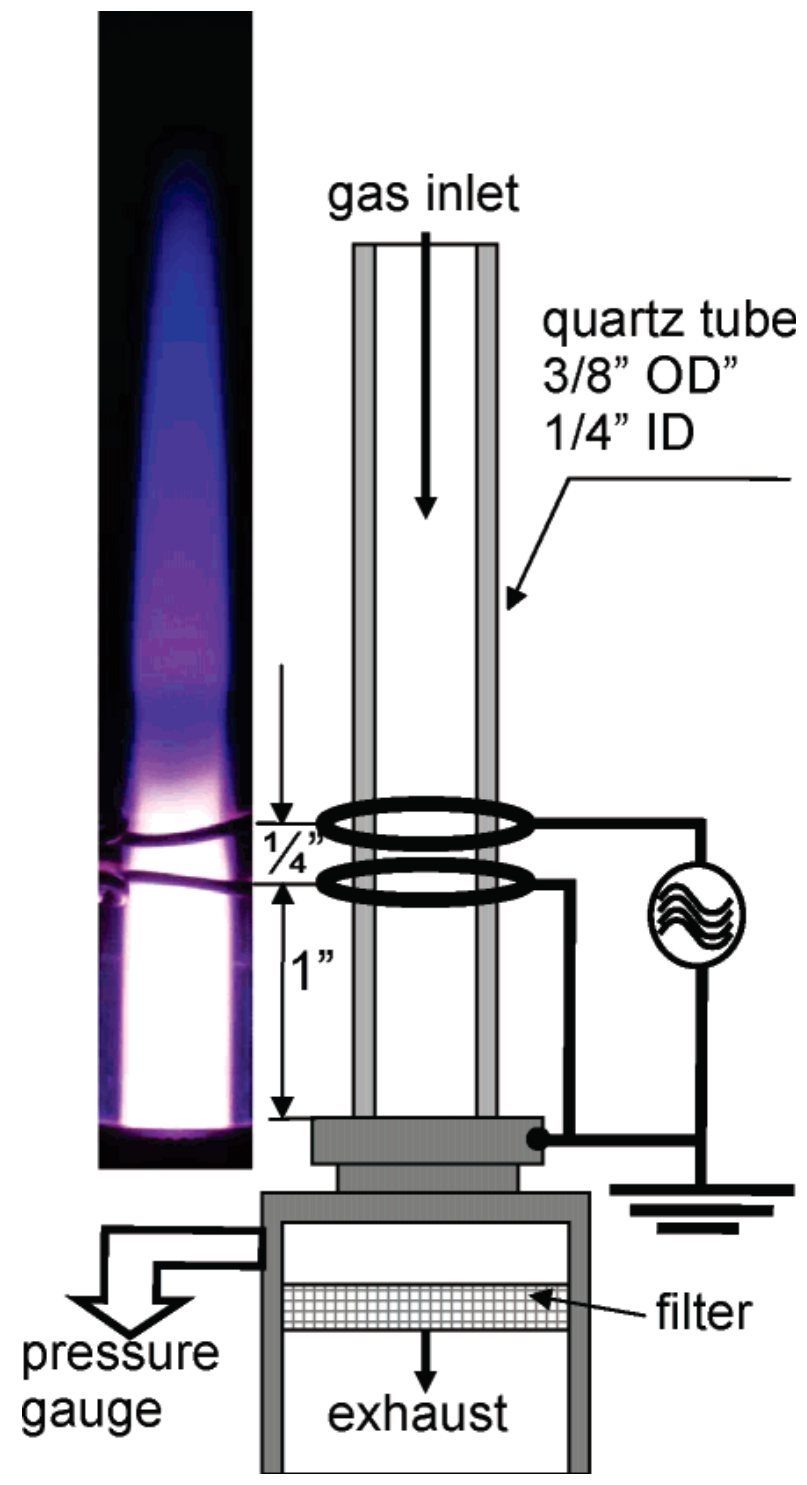


Figure 1.3 [7] UV assisted photo oxidation mechanism

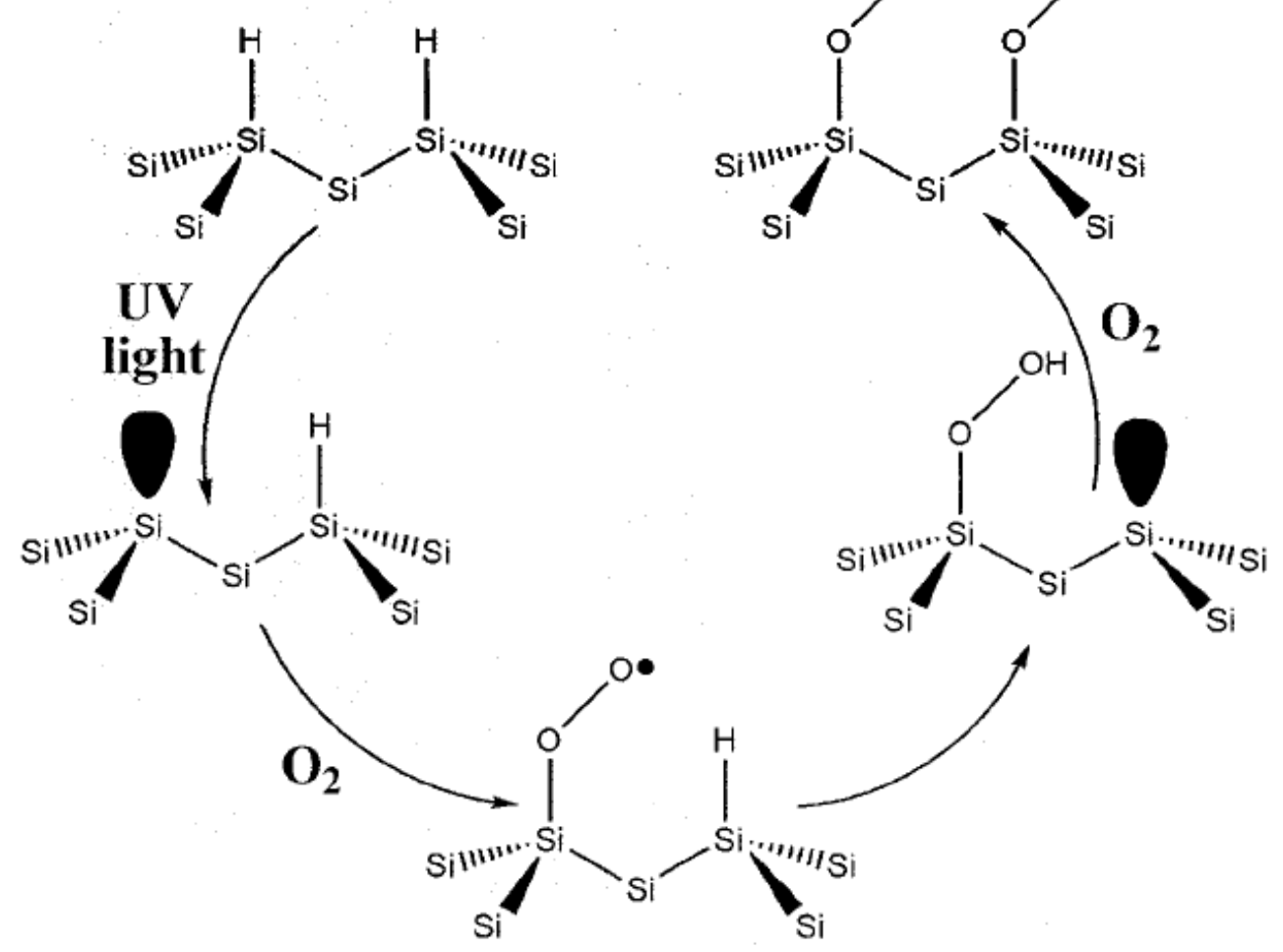


Figure 1.4: Commonly used semiconducting polymers

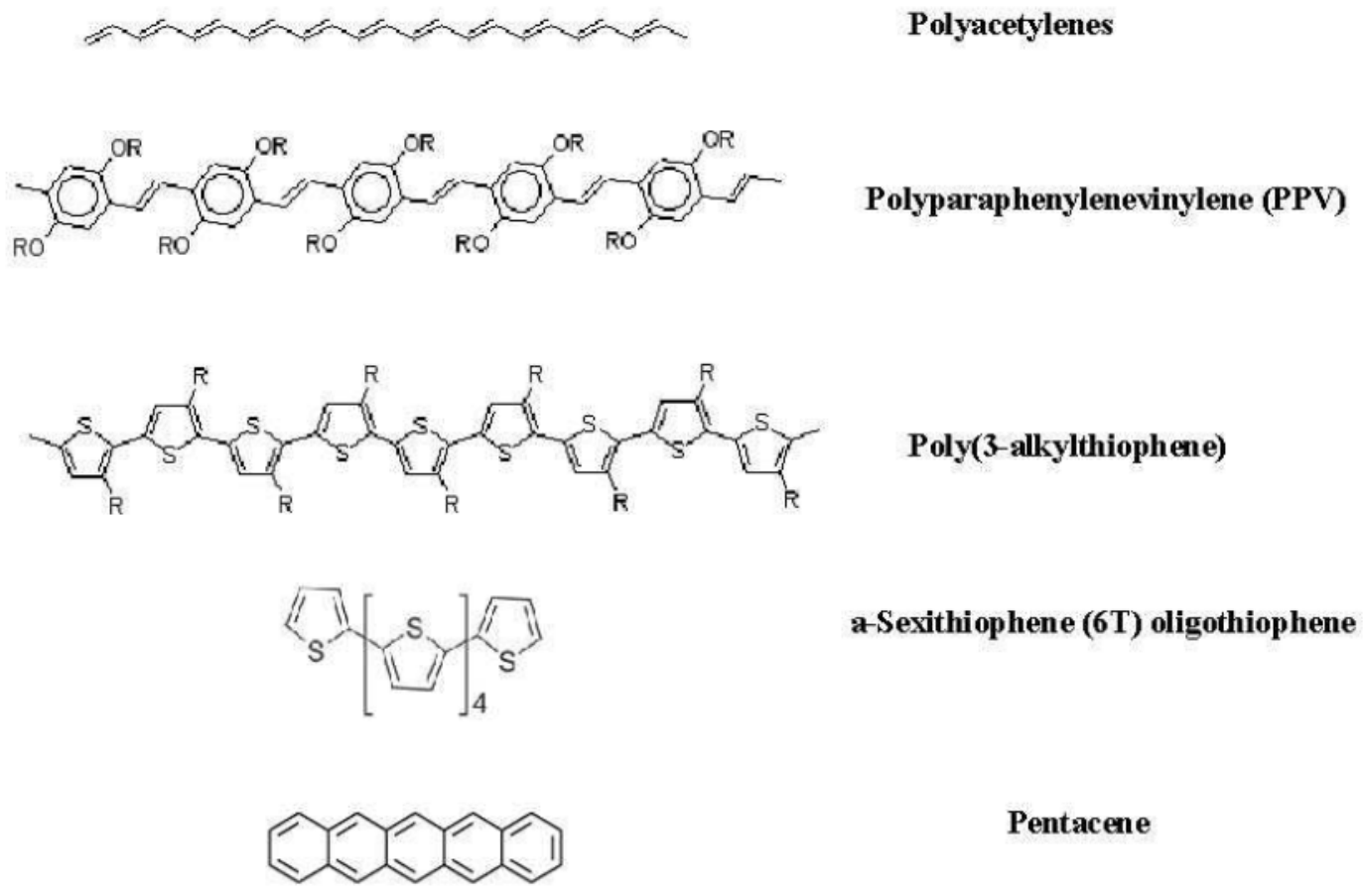

Figure 1.5: Structure of poly(3-hexylthiophene) (P3HT)

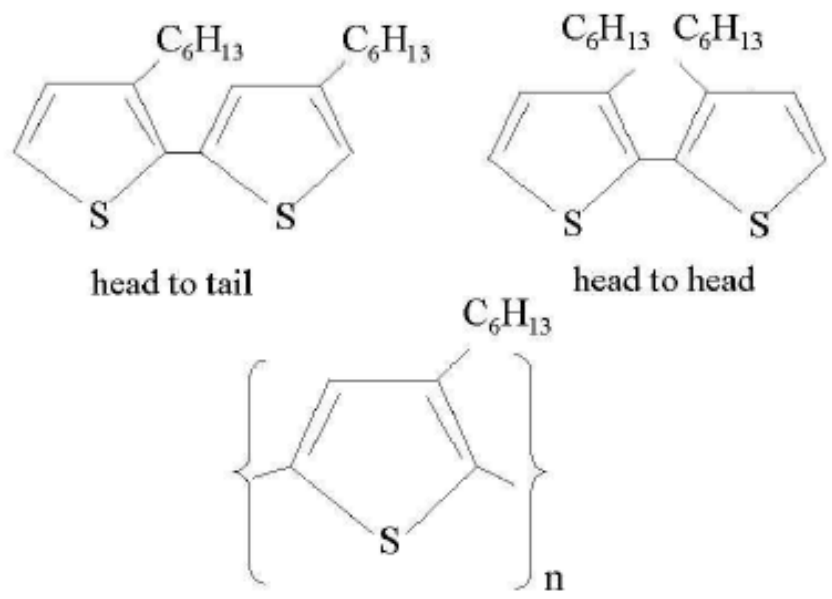


Figure 1.6: Structure of poly(3-hexylthiophene) (P3HT)

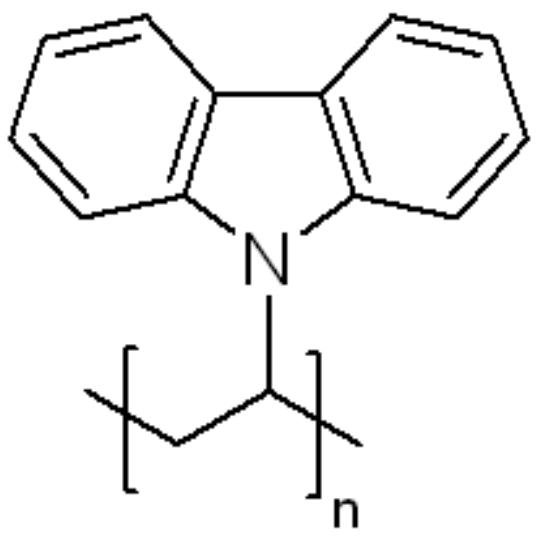

Figure 1.7 [20] First type of JFET

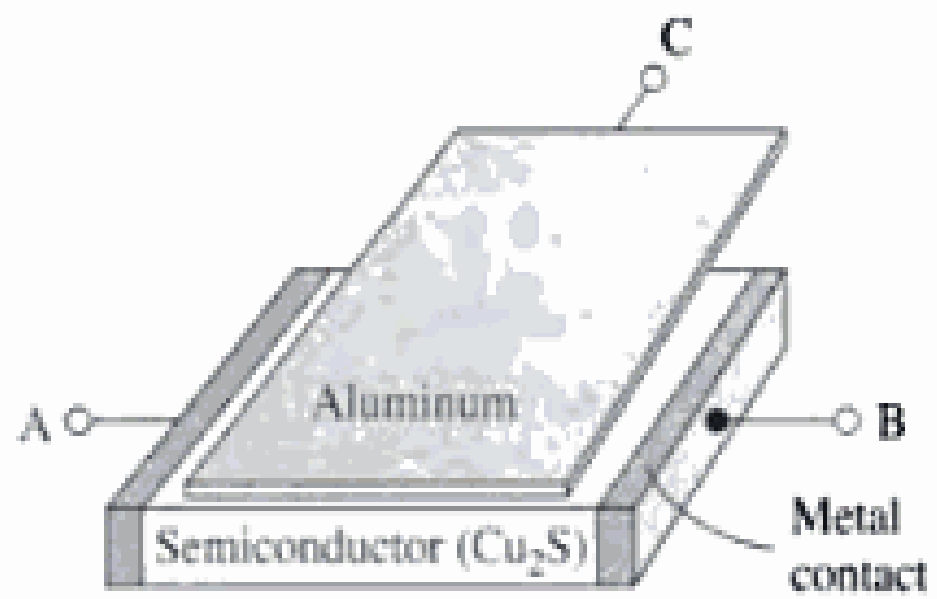


Figure 1.8 [20] Cross section of a n channel p-n junction FET

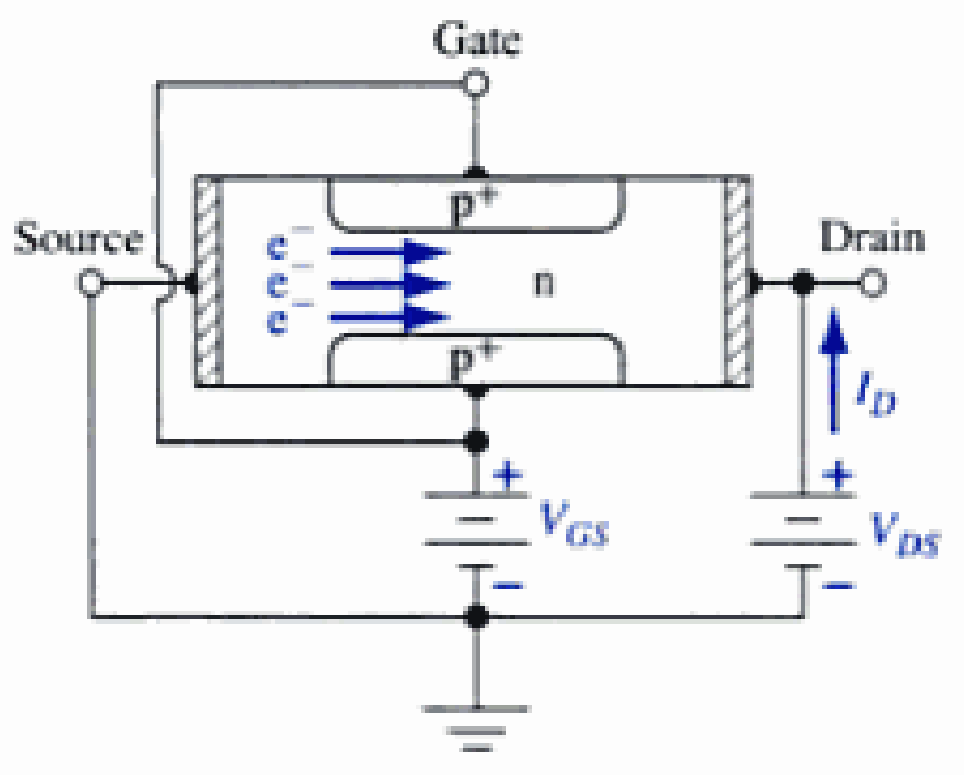


Figure 1.9: Single layer hybrid device (a) band diagram showing respective workfunction, conduction, valance, HOMO and LUMO energies for the different materials.

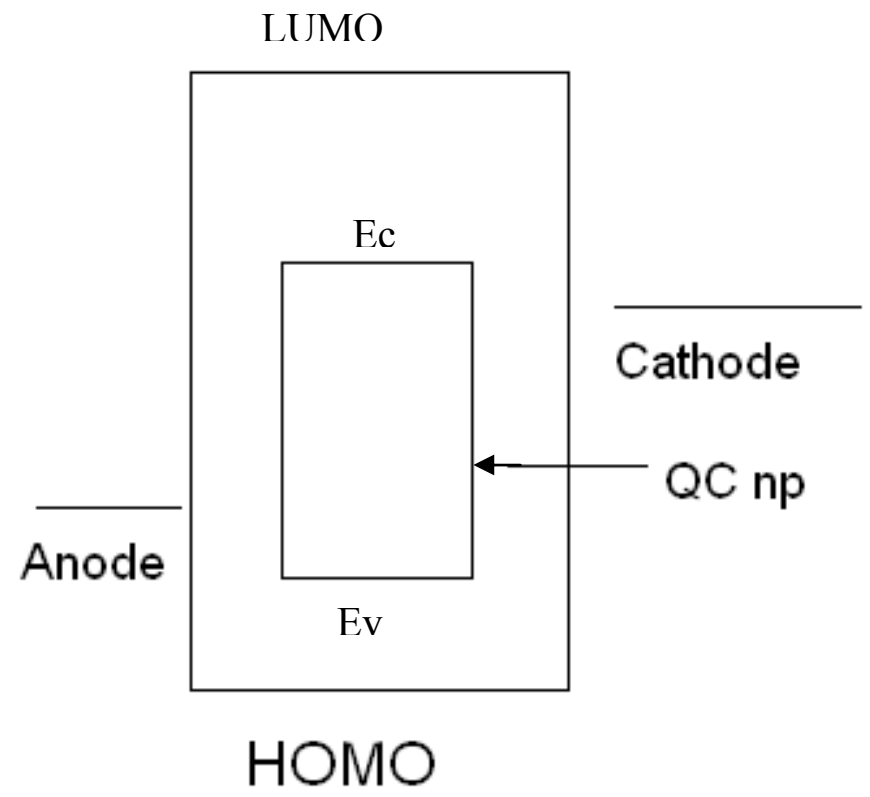


Figure 1.10: Multilayer hybrid device (a) band diagram showing the respective workfunction conduction, valance, HOMO, LUMO energies for the different materials, and (b) layout under forward bias. Within this figure EL denotes emissive layer.
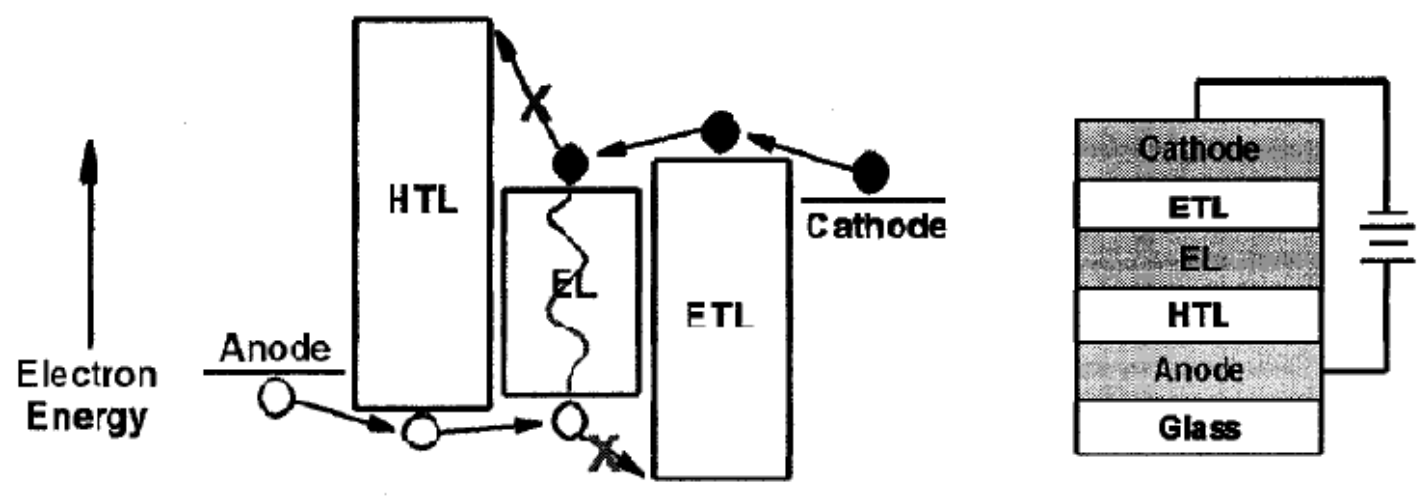


\section{Chapter 2}

\section{Production and Synthesis of Nano particles}

The production and passivation process for silicon nano particles is presented in this chapter. Particles are produced in an air-free environment and are coated with organics like dodecene. Coating with organics prevents particles from agglomeration and improves the quantum yield of the material.

\subsection{Introduction}

Science the discovery of size tunable visible emission from quantum confined porous silicon; EL devices that utilize quantum efficient nano particles have been sought. Such devices have been fabricated by incorporating silicon nano particles in different host dielectric matrices fabricated using clean room techniques [37], [38], [39], [40], [41] and [42]. These one step techniques are advantageous as they improve the silicon nano particle quantum efficiency by inherently passivating dangling surface bonds with the host matrix. These techniques require harsh processing conditions. Due to a lack of suitable conductive host matrix materials which can survive the harsh temperatures and processing conditions, it is preferred to instead utilize a three step process:

1. Produce free standing crystalline nano particles.

2. Passivate nano particles using ex-situ means.

3. Incorporate them into a conductive matrix.

A variety of techniques have been used for the synthesis of quantum dots. Wet chemistry and the inverse micelles reaction route [43], [44], [45], [46], [47] and [48], typically 
follow the reduction of $\mathrm{SiCl}_{4}$ with various agents such as sodium and lithium napthalenide and lithium aluminum hydride. The liquid phase reaction which works well for II-VI semiconductors, presents problems with silicon such as wide size distribution range and poor crystallinity.

In this work silicon nano particles are produced by passing silane through non-thermal plasma. The most obvious advantage of non-thermal plasma is less agglomeration due to unipolar charging of the nano particles in the discharge [49], [50] and [51]. This is a direct consequence of the thermal non-equilibrium between electrons and heavy species. The particle acquires a net negative charge in order to balance electron and ion fluxes to the surface, and the electrostatic repulsion between particles significantly quenches agglomeration.

Plasma produced silicon nano crystals shows weak photoluminescence right after production. The growth of about a $1 \mathrm{~nm}$ thick oxide shell improves the optical properties of the quantum dot with respect to the as produced material. Still the quantum yield of the surface oxidized material is fairly low unless something else is done.

Liquid phase passivation techniques are well known and have been applied to flat silicon surfaces [52], [53], [54] and [55] porous silicon [56] and [57], freestanding nano crystals obtained from liquid phase synthesis [58], [59] and [60] and freestanding nano crystals etched with an $\mathrm{HF} / \mathrm{HNO}_{3}$ mixture [61] and [62].

Liquid phase passivation techniques strategies have been extensively characterized by various characterization techniques, proving that the surface of the small crystallites can be efficiently functionalized with various organic molecules. Liquid phase treated 
organically capped porous silicon surfaces or silicon nano particles show intense visible photoluminescence and are fairly resistant to oxidation. In case of freestanding particles, a stable colloidal solution of silicon can be obtained. This greatly improves the liquid phase processibility of the material, which is of fundamental importance for several applications.

\subsection{Experimental Details}

\subsubsection{Silicon Nano Particles Non Thermal Plasma Fabrication and Passivation}

Silicon nano particles are fabricated in non thermal plasma through the electron impact dissociation of silane gas, leading to cluster formation of nanocrystal growth. Downstream of the plasma, nanocrystals of $6 \mathrm{~mm}$ diameter are collected in a powder form on a stainless steel mesh. The silicon nano particle coated mesh was carefully transferred under nitrogen to chloroform $\left(\mathrm{CHCl}_{3}\right)$ and ultrasonicated to create nano particle dispersion.

The as-produced nano particles do not PL due to incomplete surface passivation leaving non-radiative recombination centers. To improve the PL of these nano particles a liquid phase passivation technique is adopted. The following is the procedure for the passivation process:

1. Extraction of the produced nano crystals into an air free environment. This is done to minimize the material exposure to the oxidizing agents such as air and water and to study the effect of subsequent surface oxidation on the material properties.

A glove bag inserts the system exhaust so that the sample can be removed from 
the nitrogen atmosphere. The glove bag is purged with nitrogen for several hours before making the particles. After extraction sample is placed in a vial which is sealed with rubber septum and sample is then transferred from the glove bag.

2. The vial is then filled with the desired hydrosilating mixture. Air exposure is avoided using a Schlenk line and cannula transfer between the vials containing sample and hydrosilating mixture. Before reaction, the hydrosilating solution is kept in contact for many hours with molecular sieves, whose purpose is to remove water from the solution. High purity nitrogen is bubbled through the hydrosilation solution for many hours to remove dissolved oxygen. After refilling, the vial is pressurized with nitrogen to avoid air contamination of the sample.

3. Sonicate off the nano particles from the mesh. At this point no reaction has taken place between the reactant molecule and the particles and the mixture is not a clear colloidal dispersion.

4. Cannula transfer of the slurry obtained after sonication in to the reaction flask. In the case of a thermally activated reaction, the reactor consists of $200 \mathrm{ml}$ pyrex flask with a water cooled refluxer mounted on it. The reactor is sealed with a rubber septum. Before reaction the flask is washed with Potassium Hydroxide $(\mathrm{KOH})$ to remove any residual particles from previous batches, rinsed first with hot water, and then rinsed with alcohol and chloroform. The refluxer is mounted on the flask, and the reactor is evacuated and refilled with nitrogen using Schlenk line. The flask is heated to about $150^{\circ} \mathrm{C}$ for about 2 hours and then cooled before the reaction for the freshly prepared batch. It is extremely important to seal the 
junction between the reaction flask and the refluxer. A Teflon O-ring seems to work well for this purpose.

5. The solution is kept at its boiling point for several hours. The reactor is constantly purged with nitrogen during the reaction. The UV initiated reaction is typically slower.

6. Drying the sample. After the reaction is completed the solution is cooled down and transferred to a different flask that has been cleaned and baked with the same procedure as with the reaction flask. No refluxer is used during drying. The solution is brought to a temperature of approximately $80^{\circ} \mathrm{C}$ and nitrogen is continuously flowed into the flask. Figure 2.1 shows the chemical reaction that takes place on nanoparticles.

Many steps are involved in this passivation process and each one of them presents a potential risk of air exposure and sample oxidation. Sealing and drying of the flask is particularly important, since the sample is treated at high temperature for several hours and any defect in sealing could lead to an oxidation of silicon nanoparticles. The solution should never come in contact with the rubber septa, since they dissolve into the solvents and they also induce blue florescence when the solution is exposed to the UV source. Figure 2.2 shows the photoluminescence spectrum of dodecene coated silicon quantum dots. 
Figure 2.1: Description of Chemical Reaction on Silicon Nano Particles.
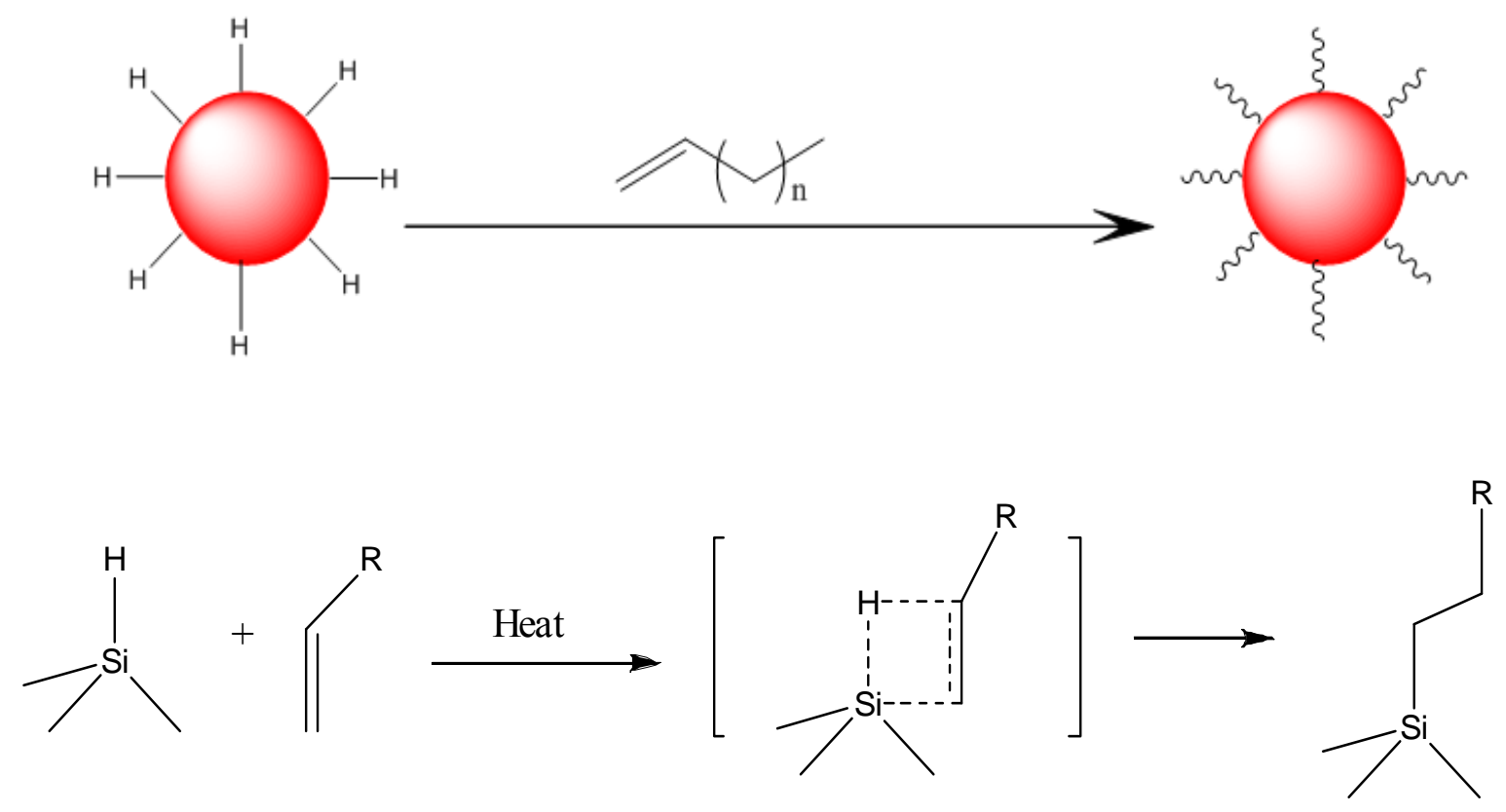
Figure 2.2: Photoluminescence Spectrum of Dodecene Coated Silicon Quantum Dots.

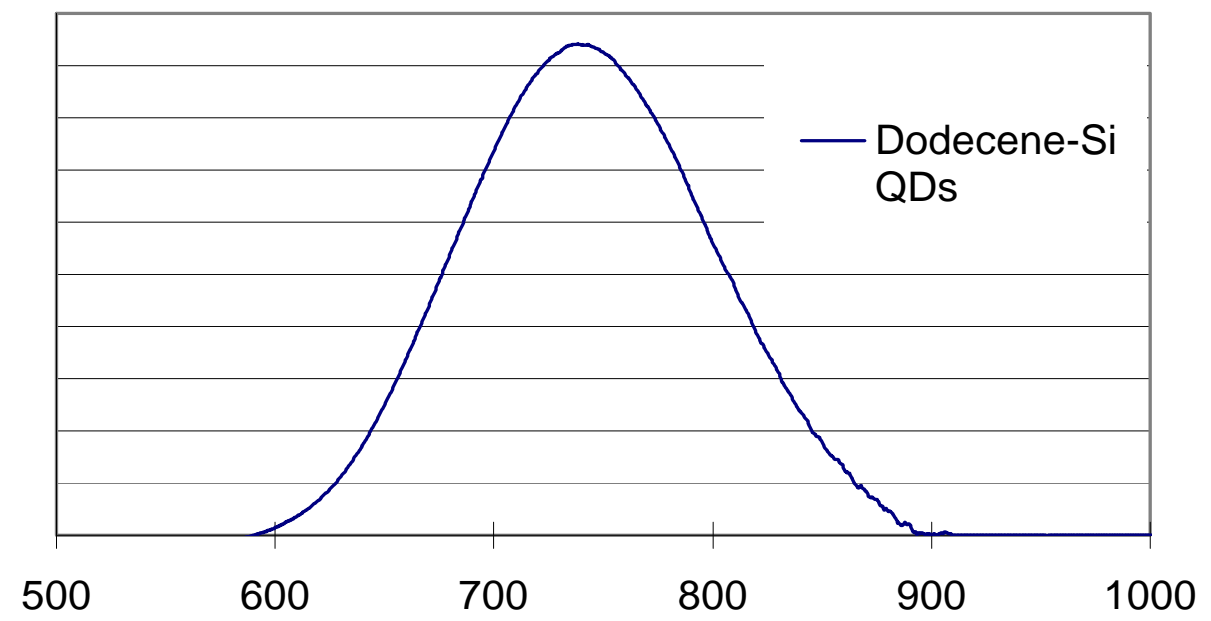




\section{Chapter 3}

\section{Conducting Polymers}

\subsection{Introduction}

Review of conducting polymers is done in this chapter. Polymers like poly $(3,4-$ ethylenedioxythiophene):poly(styrenesulfonate) commonly known as PEDOT:PSS and Poly(N-vinylcarbazole) (PVK) are used in this project. This chapter includes problems faced in making a uniform and stable film from these polymers and steps taken to overcome those problems.

Advent of computer technology over the past few decades has spurred research in many different areas. The desire to make organic based components for computers has lead to much research in the area of conjugated polymers. Organic polymers can be produced by synthesis through reactions of functional groups of monomers. In figure 3.1 repeat unit for some common conjugated polymers are shown.

Conducting Polymers are unique class of materials that have generated heightened interest over the past decade. Natta, Mazzanti and Corradni [64] converted acetylene to form a polymer polyacetylene for the first time in 1958. In 1977 H. Shirakawa and coworkers discovered that the semiconducting polyacetylene (PA) films could be made conducting by chemical doping [65]. In $1990 \mathrm{~J}$. Tsukamoto reported that the conductivity of an iodine-doped polyacetylene approaches that of a copper [66]. This is why conjugated polymers are called conducting polymers. 
The 2000 Nobel Prize was awarded to MacDirmand, Heeger and Shirakawa for their pioneering work on conjugated polymers as semiconducting materials [67], [68] and [69]. Another major step occurred in 1990 when electroluminescence was reported in poly(pphenylene vinylene) (PPV) [70]. In other words, it was shown that conjugated polymers could be used as the emitting layer in the light emitting devices (LED's). Conjugated polymers are extremely versatile and broad in their application possibilities with conducting properties, optical properties, shielding capabilities and luminescent properties. They can be used as batteries and biosensors and can be made to cover large areas (Flat Panel Displays).

In this work I have used two kind of polymers one is PEDOT:PSS which is a water based polymers and is a good conductor of holes. The other one is Poly(N-vinycarbazole) (PVK) which is used along with silicon nano particles. The polymer is mixed with nano particles and is spin coated to make a uniform layer of nano particles.

\subsection{Poly(N-vinycarbazole) (PVK)}

PVK is vinyl aromatic polymer produced by polymerization of the monomer $\mathrm{N}$ vinylcarbazole (NVK). Figure 3.2 shows both NVK and PVK. PVK was produced in 1940's [71] and [72] for the application of high temperature dielectric. In 1970, IBM Corporation introduced the first organic polymeric photoconductor based on PVK [73].

PVK is an extremely attractive material for dielectric applications because the dielectric constant $\varepsilon$ is very stable over wide temperature range. Also the bulk and surface resistivity value is high, greater than $10^{14} \mathrm{ohm} / \mathrm{cm}[74]$. 
PVK is an efficient insulator in the dark, but under UV radiation or electric field it supports the passage of positive charge carriers. Since PVK absorbs only UV radiation, it can be a true photoconductor only in that spectral region.

PVK can be useful either as an insulator or a photoconductor, depending upon the circumstances. The polymer has shown promise as a p-type semiconductor [75] though the films of the polymer exhibit loss of conductivity over the time.

\subsection{Poly(3,4-ethylenedioxythiophene):poly(styrenesulfonate) (PEDOT:PSS)}

PEDOT was discovered by scientists at Bayer AG research laboratories in Germany during the second half of the 1980's. PEDOT was initially found to be insoluble polymer and therefore hard to process [76]. However it exhibited some very interesting properties such as highly conductive, almost transparent and stable in oxidized state [77]. In order to solve the solubility process, a water soluble polyelectrolyte poly (styrenesulfonic acid) (PSS) was added as a charge balancing dopant during polymerization. [78]. The resulting PEDOT:PSS combined the properties of PEDOT and PSS, obtaining a good film-forming properties, highly conductive, high visible light transmissivity, and excellent stability. Figure 3.3 shows the monomer of PEDOT:PSS.

PEDOT-PSS is one of the most widely used materials in the field of organic electronics. PEDOT-PSS has been used to fabricate neural electrodes [79], Flexible photovoltaic's [80] as well as batteries [81].

PEDOT:PSS has been successfully used in photovoltaic and Light Emitting Devices as a hole collecting or hole conducting layer due to its high work function and electron 
blocking characteristics [82], [83] and [84]. The use of PEDOT:PSS is also desirable as it increases the lifetime of the devices [85], and in comparison with some inorganic oxide electrode, the work function of PEDOT is relatively stable, giving more reproducible device characteristics [86].

\subsection{Experimental}

Junction Field Effect Light Emitting Transistor's (JFELET) fabricated in this work, Indium Tin Oxide layer (ITO) on a glass substrate was used as the anode. ITO-coated glass slides purchased commercially were therefore used as the initial substrate.

ITO was thoroughly cleaned using ultra-sonication with acetone, methanol, isopropanol alcohol and DI water respectively for 20 minutes in each solution. These glass slides were then dried and then treated with oxygen plasma for 5 minutes. Oxygen plasma makes the ITO surface hydrophilic [87], PEDOT:PSS is a water based polymer therefore it becomes easy to spin coat the film of PEDOT:PSS on this hydrophilic surface.

PEDOT:PSS was filtered using a 0.2 micron filter to remove any agglomerates that had formed in the solution. The filtered PEDOT:PSS was then spin coated with at various spin speeds to make a film of desired thickness $(\sim 50 \mathrm{~nm})$. The slides were then dried on hot plates to evaporate any liquid contents in the films.

The PEDOT:PSS films obtained after this process were very transparent and smooth and had good adhesion with the ITO layer underneath.

The next step in the process was to pattern the aluminum gate over the PEDOT:PSS layer deposited using thermal evaporation. The thickness of aluminum was kept to $\sim 100 \mathrm{~nm}$. Liftoff was tried initially to pattern the aluminum gate. Shipley's photoresist 1813 was 
spin coated on PEDOT:PSS. PEDOT:PSS is a water based polymer and any contact of polymer with water used to make in soluble again in water. Figure 3.4 shows the layer of photoresist being washed away as soon as the samples came in contact with water during the rinsing off the remaining developer used to develop the photoresist.

I also tried to use Reactive Ion Etching of aluminum using $\mathrm{BCl}_{3}$ and chlorine gas in an argon plasma, by first depositing aluminum and them patterning photoresist over it and eventually etching the aluminum.

To etch aluminum the RIE chamber was first cleaned using an oxygen plasma and then $\mathrm{BCl}_{3}$ and $\mathrm{Cl}_{2}$ gasses were introduced in the chamber at $30 \mathrm{sccm}$ and $15 \mathrm{sccm}$ flow rates to create a pressure of 200 mtorr in the chamber to etch native oxide layer. Aluminum oxides etches in about one minute. Once the oxide is etched away the chamber is evacuated and $\mathrm{BCl}_{3}$ and $\mathrm{Cl}_{2}$ gasses are again introduced at 30 and $8 \mathrm{sccm}$ respectively at 25 Watts of power. The aluminum layer was completely etched in approximately 4 minutes.

This method of patterning aluminum also did not produced the desired results and as the deposited aluminum was porous and water would seep through it washing away the PEDOT:PSS.

To overcome this problem we used another polymer called polyepoxysilane along with PEDOT:PSS. This polymer combines with the chains of PEDOT:PSS that react with water and hence PEDOT:PSS becomes insoluble in water. This polymer was mixed at the ratio of $1 \%$ by volume in to PEDOT:PSS. The solution was then ultrasonicated for 30 minutes so that polyepoxysilane completely cross links with PEDOT:PSS. After mixing 
this polymer with PEDOT:PSS it was spin coated to form a 40nm thick film. After spin coating a layer of polymer, aluminum was deposited and etched using RIE as mentioned above. Figure 3.5 shows the patterned aluminum after reactive ion etching.

\subsection{Summary}

Since polymers are cheap and easy to reshape, they are promising materials for future devices. Basic understanding of the chemical and physics process in polymer is necessary before using them in the devices. In this project spin coating procedure for these polymers was demonstrated. Most of the conducting polymers that are available have to be treated in several different ways to obtain stable and functional films. It is important to understand the chemical properties of polymer so that any curing process used does not affect the electrical and optical properties of the polymer and the polymers can be used much more efficiently in the devices. 
<smiles>C=C/C=C/C=C/C=C/C</smiles>

a) trans-polyacetylene (PA)<smiles>Cc1ccc(C)s1</smiles>

c) polythiophene

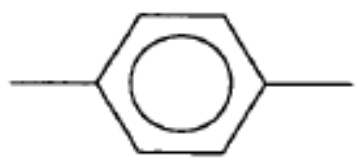

e) polyparaphenylene<smiles>[R]C(C#CC(C)(C)P)=C(C)C</smiles>

b) polydiacetylene (PDA)

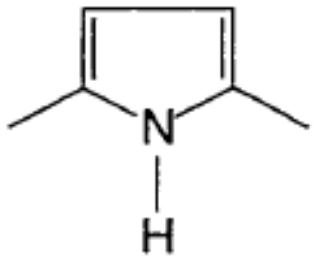

d) polypyrrole

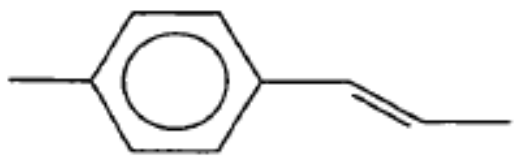

f) polyparaphenylene vinylene (PPV) 
Figure 3.2: N-vinalcarbazole and Poly(N-vinycarbazole)
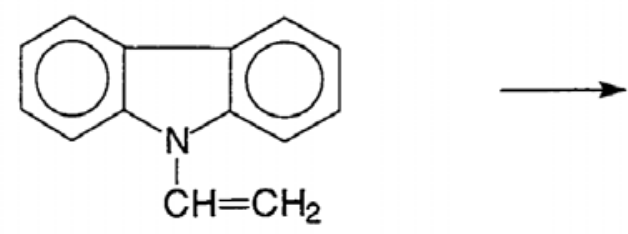

NVK

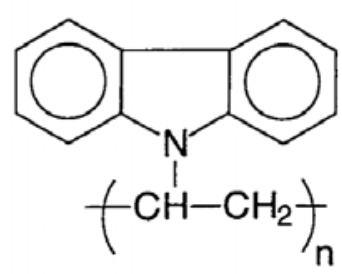

PVK

Figure 3.3: Poly(3,4-ethylenedioxythiophene):poly(styrenesulfonate) (PEDOT:PSS)

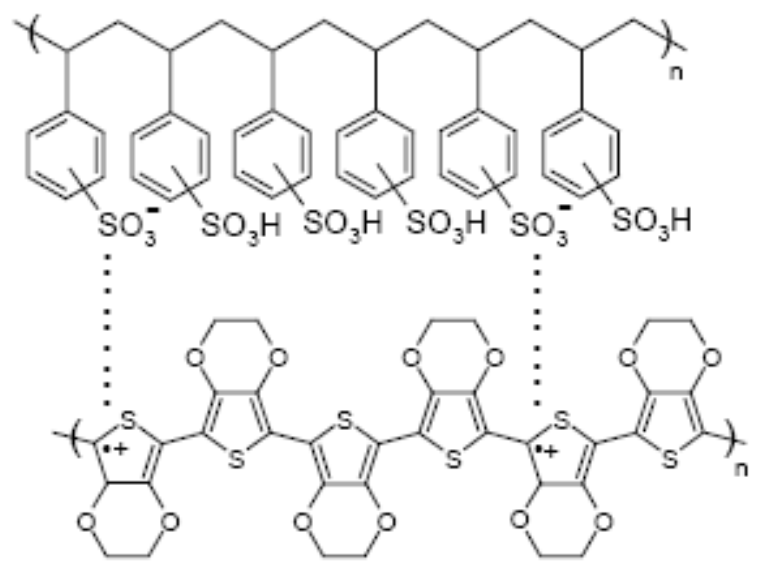


Figure 3.4: Photoresist Pattern Peeled off as PEDOT:PSS film is washed away

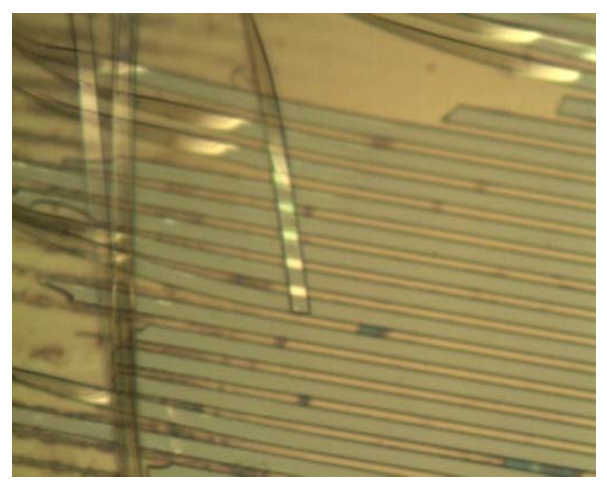

Figure 3.5: Patterned Gate over PEDOT:PSS

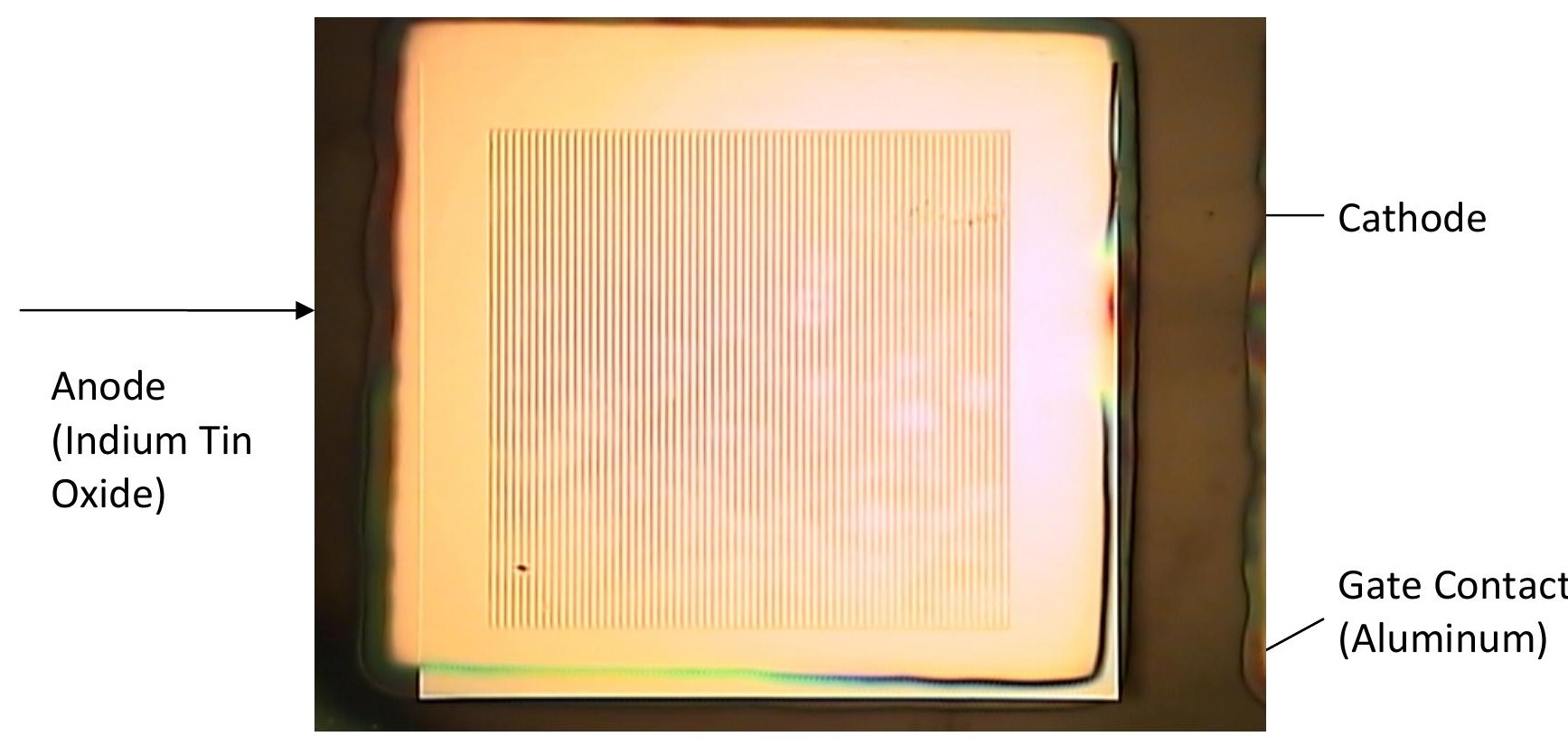




\section{Chapter 4}

\section{Hybrid Light Emitting Transistors}

\subsection{Introduction}

The physics of the Field Effect Transistor (FET) and its fabrication steps are discussed in this chapter. The concept of the field effect phenomenon was the basis for the first proposed solid state transistor. The phenomenon of modulating the conductance of a semiconductor by an electric field applied perpendicular to the surface of a semiconductor is called the field effect.

\subsection{Metal Semiconductor Field Effect Transistor (MESFET)}

There are the two general categories of Junction Field Effect Transistor (JFET). The first uses a pn junction as the control electrode or gate (JFET or pn JFET). The second one is the MEtal-Semiconductor Field Effect Transistor (MESFET) which uses a Schottky barrier rectifying junction as the control electrode. The current in the Junction Field Effect Transistor is through a semiconductor region known as the channel, with ohmic contacts at each end. Depending upon the carrier type in the on-state it is either called a $p$ channel JFET where the majority carriers from source to drain are holes or an $\mathrm{n}$ channel JFET where majority carriers from source to drain are electrons.

The basic transistor action is the modulation of the channel conductance by an electric field that is perpendicular to the channel. The modulating electric field is induced in the space charge region of a reverse biased pn junction or a reverse biased Schottky barrier 
junction. The modulation of the quasi-nuetral channel width by the gate voltage modulates the channel current.

I fabricated a P channel MESFET for my project where I used polymers for the conduction of holes and electrons and aluminum for the gate, creating Schottky barrier rectifying junction at the interface.

In the MESFET as shown in figure 4.1 the reverse bias gate to source voltage induces a space charge region under the metal gate which modulates the channel conductance. The space charge region will eventually reach the substrate if the applied negative voltage is sufficiently large. This condition is known as pinchoff.

If we treat the semi-insulating substrate as an intrinsic material, then the energy band diagram of the substrate-channel-metal is as shown in the figure 4.2 for the case of zero applied bias to the gate

There is a potential barrier between the channel and the substrate and between the channel and the metal. Therefore, majority carriers electrons are confined to the channel region.

Consider, now, another type of MESFET in which the channel is pinched off even at $V_{\mathrm{GS}}$ $=0$. Figure 4.3(a) shows this condition in which the channel thickness is smaller than the zero biased space charge width. To open a channel, the depletion region must be reduced: A forward bias voltage must be applied to the gate semiconductor junction. When a slightly forward bias voltage is applied the depletion region just extends through the channel, a condition known as threshold shown in figure 4.3(b). The threshold voltage is a gate-to-source voltage that must be applied to create the pinchoff condition. The 
threshold voltage for this n-channel MESFET is positive, in contrast to the negative voltage to the n-channel depletion mode device. If a larger forward bias is applied, the channel region opens as shown in the figure 4.3(c). The applied forward-bias gate voltage is limited to a few tenths of a volt before there is significant gate current. The device is known as n-channel enhancement mode MESFET. The advantage of enhancement mode MESFET is that the circuits can be designed in which the voltage polarity on the gate and drain is the same. However, the output voltage swing will be quite small with these devices.

\subsection{Experimental}

After patterning the aluminum gate electrodes using reactive ion etching, another layer of PEDOT: PSS was spin coated over it, as shown in figure 4.4. After spin coating PEDOT:PSS a layer of nano particles dispersed in PVK was spin coated. PVK was supplied in a solid form and so is dissolved at $10 \mathrm{mg} / \mathrm{ml}$ in chloroform. Nano particles were also dispersed in the same solution at the ratio $3 \mathrm{mg} / \mathrm{ml}$ of chloroform. The nano particle solution was spin coated at $2500 \mathrm{rpm}$ for 90 second. Devices were then kept in oven under nitrogen at $100^{\circ} \mathrm{C}$ for 30 minutes. Once all the solvent was evaporated, the top contact of aluminum was deposited using thermal evaporation. The thickness of the top contact was 100 nanometers. Devices were then treated again in an oxygen plasma for a couple of minutes to etch away polymer from the sides of the gates deposited during spin coating as shown in figure 4.5. Etching was done to prevent any leakage paths for current along the side of the devices. Figure 4.6 shows the final device. The devices 
were then tested for their electrical and optical characteristics using an Agilent 4156 device parameter analyzer and a silicon photodiode.

\subsection{Results and Discussion}

Devices were tested for optical and electrical characteristics and results were also verified using the Padre Device Simulator. I-V characteristics as shown in figure 4.7 shows the transistor characteristics at different gate bias voltage. We can see the shift in the current at as the gate voltages changes from 1volts to -3 volts. Drain current increases as the gate voltage bias becomes more and more negative.

The quantum dots electroluminesced at the same wavelength as the photoluminescence, suggesting little blue shift caused by processing. The light intensity increased with gate bias as more charge was injected into the polymer, but reached a maximum when the depletion regions from adjacent gate electrodes pinched off the channel. The maximum light output from the device was typically found to occur at about $\mathrm{Vg}=-5$ volts. Similar characteristics were recorded when the devices were tested for electrical characteristics. Figure 4.8 shows the optical output of the device as the gate bias decreases from -2volts to -6 volts. We can see increase in the light output from the device as the gate bias decreases from -4 volts to -5 volts. Light was also seen a higher gate bias at -3 and -2 volts but it was significantly less than what is seen at -5 volts, which suggest that there is a maximum in the rate of combination of electron and holes producing photons at this bias. As the gate bias is decreased further we see a decrease in the light output. 
Figure 4.9 shows the simulations graphs obtained by Padre Simulator. Simulations were done using silicon as material for the device with some properties (doping) modified to make it similar to polymers.

Simulations results obtained are qualitatively similar to the experimental results. Silicon was assumed as the channel material; this led to much higher currents than those observed experimentally. Thia was attributed to the higher mobility in silicon. Accurate values for the conductivity of the polymers used here is unknown as it is highly process dependent.

\subsection{Conclusions and Future Work}

A Hybrid Light Emitting Metal-Semiconductor Field Effect Transistor was fabricated using silicon quantum dots and was tested for electrical and optical characteristics. We could modulate light from the device by controlling the gate bias. Light output was a maximum at -5 volts of gate bias. This suggests that the maximum recombination took place at this bias.

\subsection{Project Conclusions}

As the demand on our world's energy continue to grow, alternative high efficiency materials such as quantum confined Si nano particles are becoming desirable for their potential low cost applications in white light illumination, in optical displays and in on chip optical interconnects. Many different silicon nano particle fabrication and passivation techniques have been developed that produce silicon nano particles with high photogenerated quantum yield. However, the demonstration of high electroluminescent 
efficiency has eluded our society. This may be due to a lack of stable surface passivation layer and a device fabrication technique that can be used to construct devices around the silicon nano particles while preserving their optical properties.

Silicon nano particles were treated with dodecene as a capping layer to prevent them from oxidation. Dodecene has 10 carbon chains and it is believed if it could be reduced to 8 or 6 carbon chain then more light could be produced and efficiency of the device can be increased. New carbon chains were tried but not all nano particles could be capped with it and we could see agglomerates. Other ideas need to be investigated to carry out the reaction at higher temperature so that the total reaction time can be reduced and all the particles could be capped before they form an oxide layer over them.

Also new solvents and organics can be tried so that it is easy to load a high concentration of nano particles and at the same time be able to spin coat a smooth thin film. PVK is a hole conducting polymer and it is believed that there are charge paths through PVK instead through nano particles which decreased the efficiency of the devices. Higher band gap materials like PMMA [89] etc. need to be studied more so that the nano particles can be spin coated along with them. 
Figure 4.1 [88]: Cross section of a N channel MEsfet with semi-insulating substrate

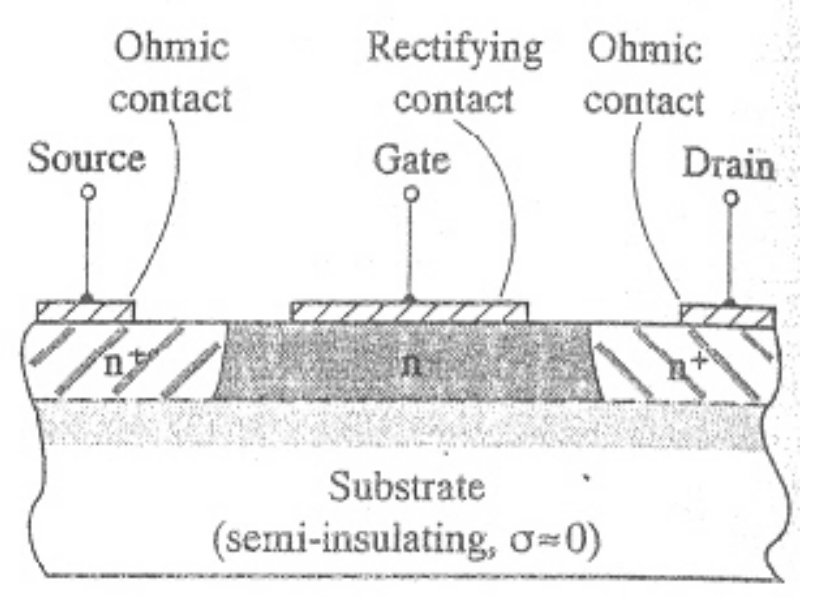

Figure 4.2 [88]: Idealized Energy band Diagram of the substrate- channel- metal in the n-channel MESFET
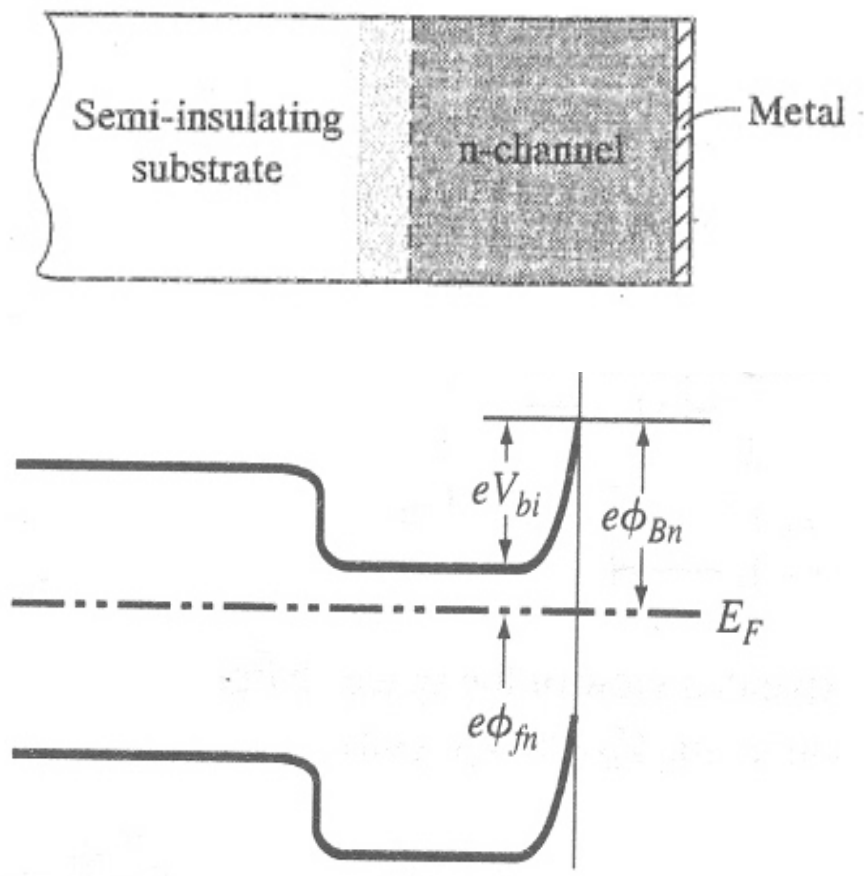
Figure 4.3 [88]: Channel space charge region of an enhancement mode MESFET for (a) VGS=0, (b) VGS = VT (c) VGS > VT

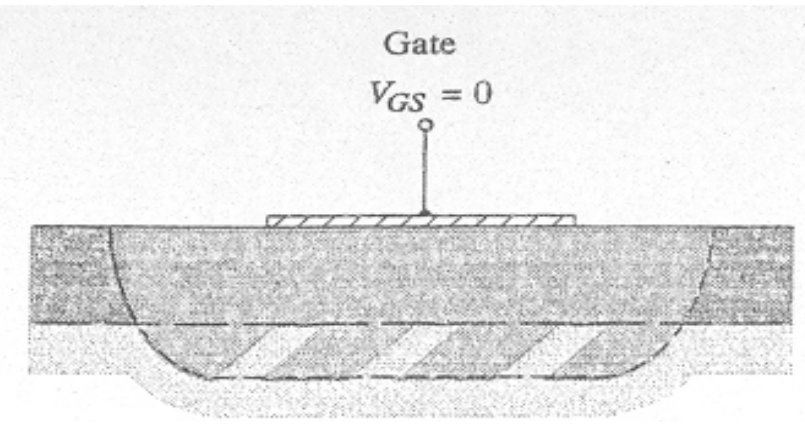

(a) n-channel

Semi-insulating substrate

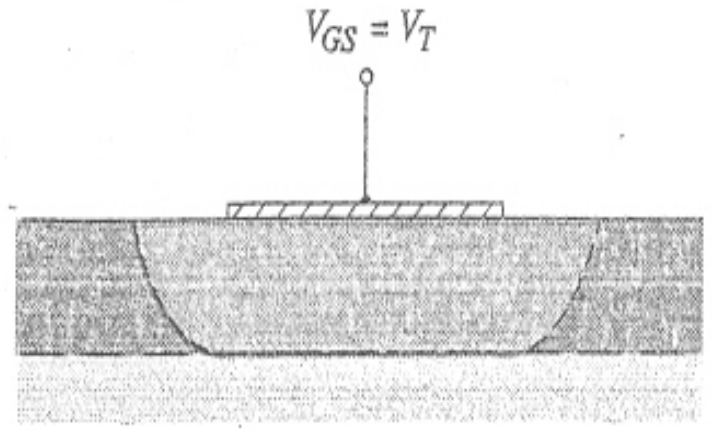

(b)

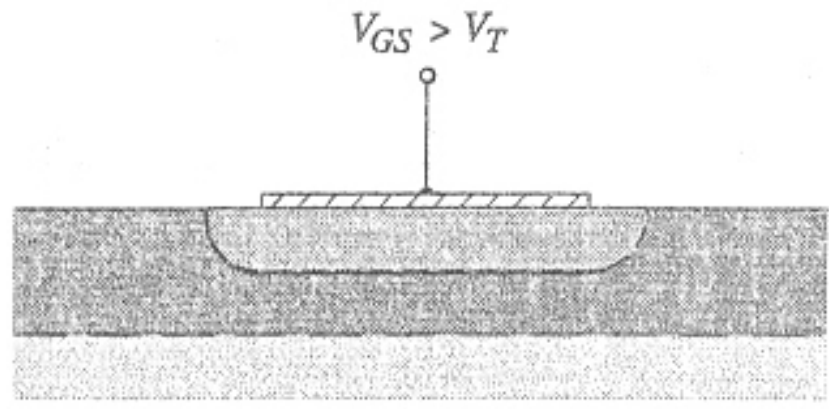

(c) 
Figure 4.4: PEDOT:PSS is spin coated over Aluminum Gates

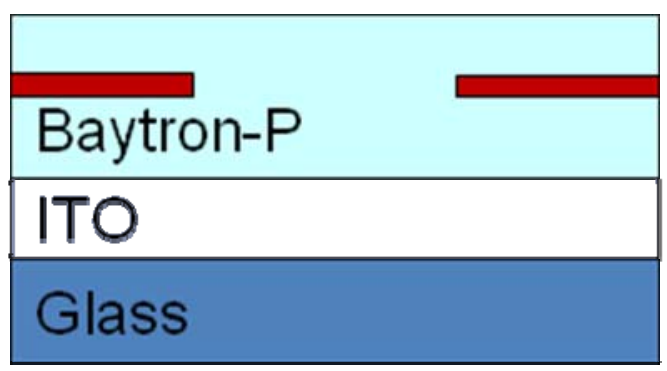

Figure 4.5: Device after spin coating nano particles and depositing top aluminum electrode

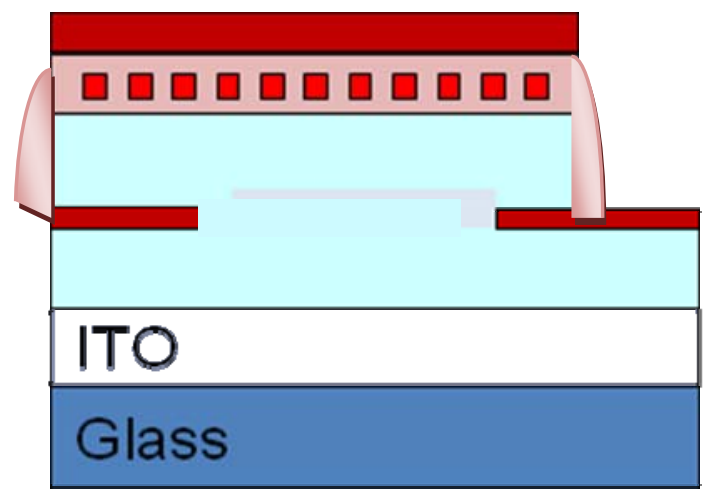


Figure 4.6: Final Device

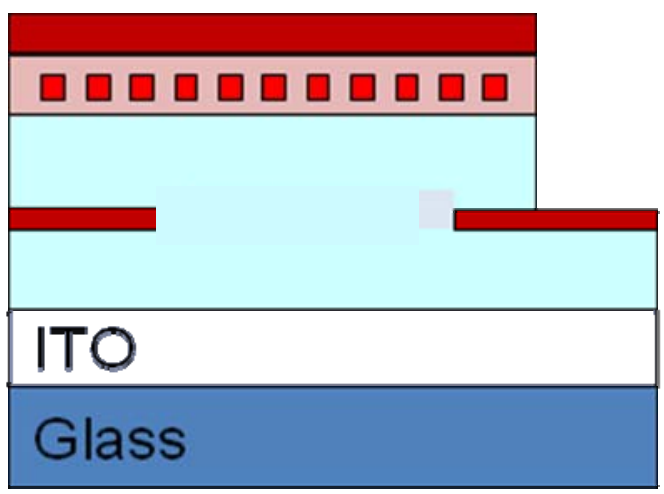

Figure 4.7: Electrical Characteristic of the devices
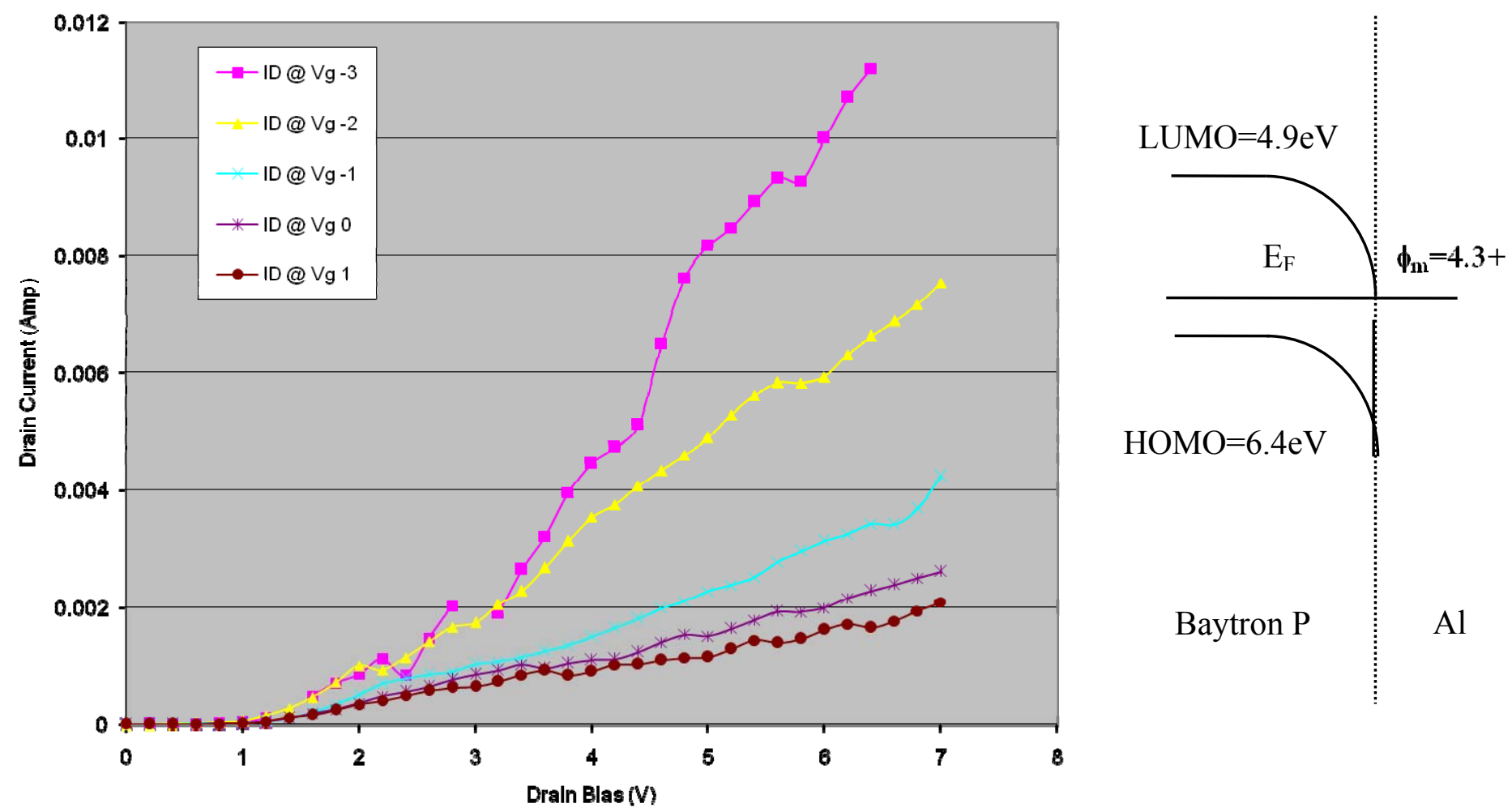
Figure 4.8: Optical characteristics of the device

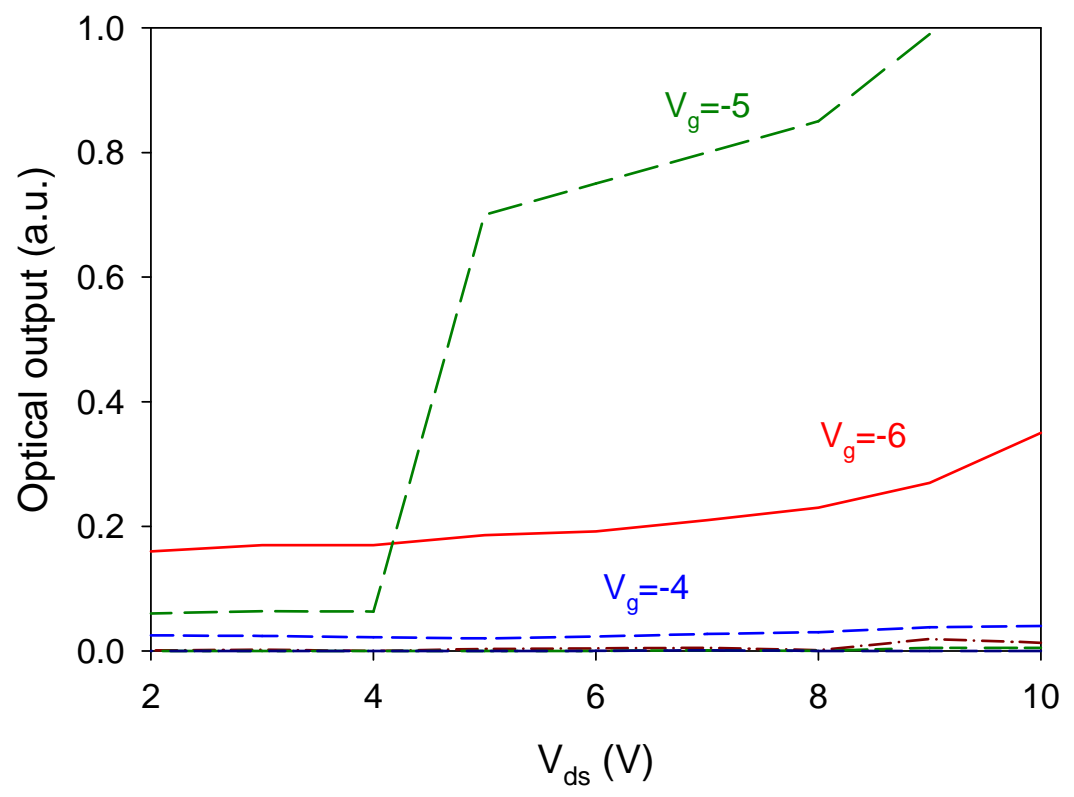

Figure 4.9: Simulations results by using PADRE simulator

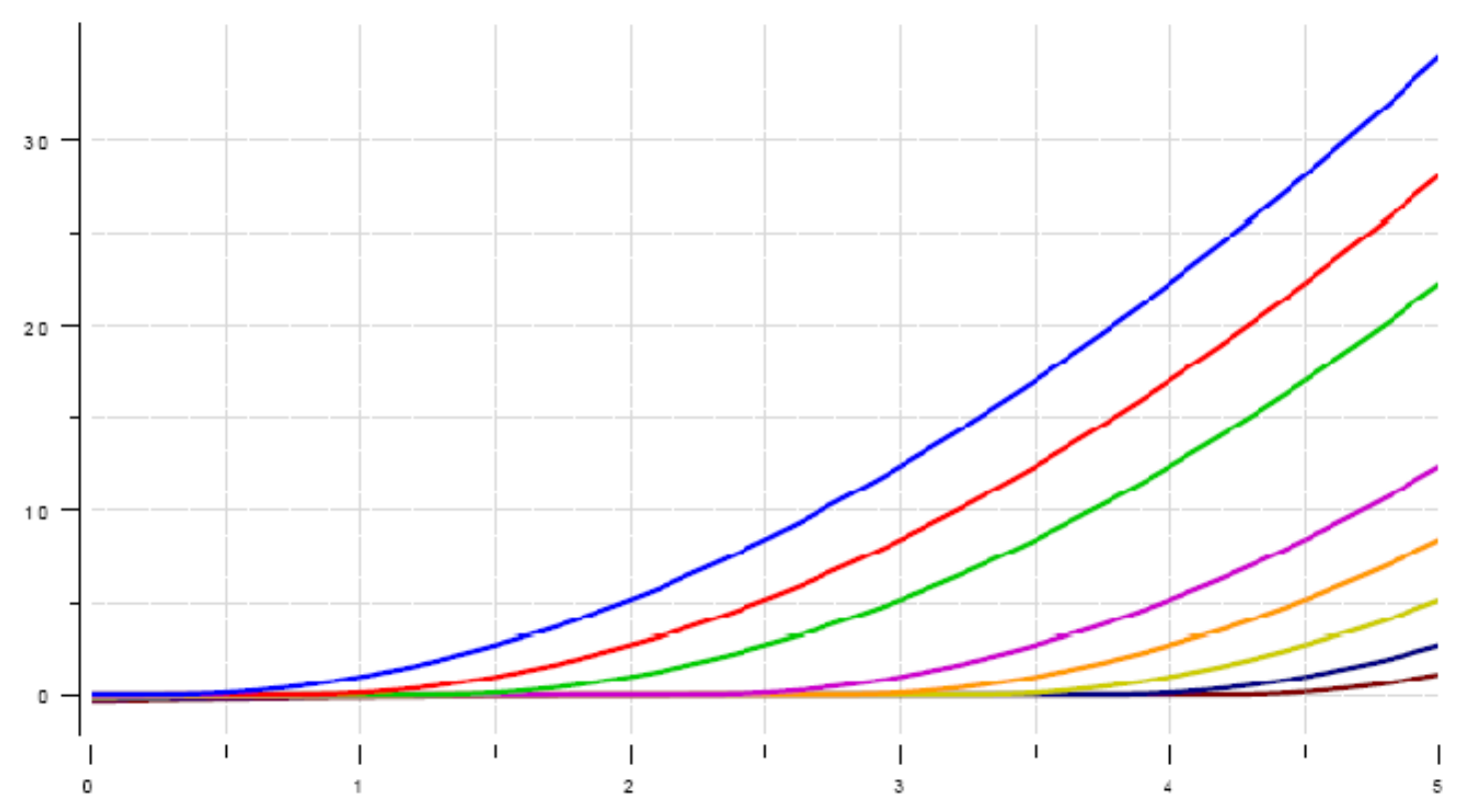




\section{References}

1. E. Werwa, A.A. Seraphin, L.A. Chiu, C. Zhou, K.D. Kolenbrander, Applied Physics Letters, Volume 64 (1994) page 1821.

2. H. Mizuno, H. Koyama, N. Koshida, Thin Solid Films Volume 297 (1997), pages 61 .

3. K.Murakami, T.Makimura, N. Ono, T. Sakuramoto, A.Miyashita, O. Yoda, Applied Surf. Science, Volume 127 (1998) page 368.

4. L. Patrone, D. Nelson, V. Safarov, M. Sentis, W. Marine, J. Lumin. Volume 80, (1999) pages 217.

5. Sykora, M.; Mangolini, L.; Schaller, R.D.; Kortshagen, U.; Jurbergs, D.; Klimov, V.I. Size-dependent intrinsic radiative decay rates of silicon nanocrystals at large confinement energies, Physical Review Letters, v 100, n 6, 15 Feb. 2008, 067401$1-4$

6. Ligman, R.K, Mangolini, L., Kortshagen, U.R., Campbell, S.A., Electroluminescence from surface oxidized silicon nanoparticles dispersed within a polymer matrix, Applied Physics Letters, Volume 90, No 6, 5 February. 2007.

7. M.V Wolkin, J. Jorne, P. M. Fauchet, G. Allan, and C. Delerue, " Electronic states and Luminescence in porous silicon quantum dots: The role of oxygen", volume 82, 1998, page 197.

8. F. Reboredo and G. Galli, "Theory of alkyl-terminated silicon quantum dots", Journal of Physical Chemistry B, volume 109, 2005, pages 1072.

9. J. Heitmann, F. Muller, M. Zacharias and U Gosele, "Silicon Nanocrystals: size matters", Advanced Materials, volume 17 page-795, 2005.

10. P. Photopoulos and A. Nassipolulou, "Room- and low -temperature voltage tunable electroluminescence from a single layer of silicon quantum dots in between two thin SiO2 layers", Applied Physics Letters, Volume 77, page-1816, 2000 .

11. C. Lui, C. Li, A. Ji, L. Ma, Y Wang, and Z. Cao, “ Exploring extreme particle density and size of blue photoluminescence from as-deposited amorphous siliconin SiNx films", Applied Physics Letters, Volume 86, page-223111, 2005.

12. F. Lacona, G. Franzo, and C. Spinella, " correlation between luminescence and structural properties of silicon nanocrystal", Journal of Applied Physics, Volume87, page 1295, 2000.

13. F. Gutmann and L. E. Lyons, Organic Semiconductors, John Wiley \& Sons, Incorporations, 1967.

14. W. F. Pasveer, Charge and Energy Transport in Disordered $\pi$-conjugated Systems, $\mathrm{PhD}$ thesis, Technische Universiteit Eindhoven, 2004.

15. F. Garnier, Scope and limits of organic-based thin-film transistors, Philosophical Transactions of the Royal Society London, Series A (Mathematical, Physical and Engineering Sciences), v 355, n 1725, 15 April 1997, p 815-27,

16. J. M. Shaw and P. F. Seidler, "Organic electronics: Introduction," IBM J. Res. Dev., vol. 45, pp. 3-9, 2001.

17. E. Cantatore, "Organic materials: A new chance for electronics?," Proceedings of the SAFE/IEEE workshop, vol. workshop 27, pp. 27-31, 2000. 
18. C. D. Dimitrakopoulos and D. J. Mascaro, "Organic thin-film transistors, a review of recent advances," IBM J. Res. Dev., vol. 45, pp. 11-27, 2001.

19. B. Dabbousi, M. Bawendi, O. Onitsuka, and M. Rubner, "Electroluminescence from CdSe quantum-dot/polymer composites”, Applied Physics Letters, vol. 66, pp.1316, 1995.

20. V. Colvin, M. Schlamp, and A. Alivisatos, "Light -emitting diodes made from cadmium salenide nano crystals and a semiconducting polymer", Nature, vol. 370, pp. 354, 1995.

21. M. C. Schlamp, X. Peng, and A. P. Alivisatos, "Improved efficiencies in Light emitting diodes made with $\mathrm{CdSe}(\mathrm{CdS})$ core/shell type nano crystals and a semiconducting polymer" Journal of Applied Physics, vol. 82, pp.5837, 1997.

22. S. Coe, W. Woo, M. Bawendi, and V. Bulovic, "Electroluminescence from single mono layers of nano crystals in molecular organic devices", Nature, vol.420, pp. 800, 2002.

23. J. Zhao, H. Zhang, C. Jiang, J. Bohnenberger, T. Basche, and A. Mews, "Electroluminescence from isolated $\mathrm{CdSe} / \mathrm{ZnS}$ quantum dots in multilayer light emitting diodes", Journal of Applied Physics, vol. 96, pp.3206, 2004.

24. J. H. Park, J. Y. Kim, B. D. Chin, Y. C. Kim, J. K. Kim and O. O. Park, "White emission from polymer/quantum dot ternary nano composites by incomplete energy transfer", Nanotechnology, vol. 15, pp. 1217, 2004.

25. Y. Li, A. Rizzo, M. Mazzeo, L. Carbone, L. Manna, R. Cingolani, and G. Gigli, "White organic Light emitting devices with $\mathrm{CdSe} / \mathrm{ZnS}$ quantum dots as a red emitter", vol. 97 pp113501-1, 2005.

26. J. Kido, H. Shionoya, and K. Nagai, "Single layer white light emitting organic electroluminescent devices based on dye dispersed poly(N-vinylcarbazole)", Applied Physics Letters, vol. 67, pp. 2281, 1995.

27. H. Mattoussi, L. H. Radzilowski, B. O. Dabbousi, E. L. Thomas, M. G. Bawendi, and M. F. Rubner, " Electroluminescence from hetero structures of poly(phenylene-vinylene) and inorganic CdSe nano crystals", Journal of Applied Physics, vol. 83, pp. 7965, 1998.

28. K. S. Narayan, A. G. Manoj, J. Nanda and D. D Sarma, "Dual function hybrid polymer nano particles devices", Applied Physics Letters, vol. 74, pp. 871, 1999.

29. G. Pfister, "Hopping transport in a molecularly doped organic polymer", Physical Review B, vol. 16, pp. 3676, 1977.

30. Y. Zhang, Y. Hu, J. Chen, Q. Zhou, and D. Ma, "Charge carriers injection and transport in PVK: Alq3 blend film", Journal of Physics D: Applied Physics, vol. 36, pp. 2006, 2003.

31. C. W. Tang and S. A. VanSlyKe, "Organic electroluminescent diodes" Applied Physics Letters, vol. 51, pp. 913, 1987.

32. Donald. A. Neamen, Semiconductor Devices and Physics ( $3^{\text {rd }}$ Edition). The Mc Graw Hill Company, 2007

33. Liao. S. Y. Microwave Solid State Devices, Ebglewood Cliffs, NJ: Prentice Hall, 1985. 
34. Pierret. R.F, Field Effect Devices, Volume 4, of the Modular Series on Solid State Devices, $2^{\text {nd }}$ Edition, Reading, MA: Addison-Wesley, 1990.

35. Gagan Aggarwal, Xiaodong Pi, Ryan Lu, Ayax Ramirez, Uwe Kortshagen, and Stephen A. Campbell, Light Emitting Transistors using Silicon Quantum Dots in an Organic Matrix, 4th International Conference on Group Four Photonics Workshop, IEEE/LEOS, Leos (2008).

36. Lorenzo Mangolini, Non Thermal Plasma Synthesis and Passivation of Luminescent Silicon Nano Crystals, Dissertation Thesis, January 2007.

37. O. Jambios, H. Rinnert, X. D eVaux, and M. Vergant, "Photoluminescence and electroluminescence of size controlled silicon nano crystallites embedded in $\mathrm{SiO}_{2}$ thin films", Journal of Applied Physics, Volume 98, pages 046105, 2005.

38. C. Lui, C. Li, A. Ji, L. Ma, Y. Wang and Z. Cao, "Expolring Extreme particle density and size of blue photoluminescence from as-deposited amorphous Si-inSiNx films" Applied Physics Letters, Volume 86, Pages 223111, 2005.

39. S. Hazra, I. Sakata, M. Yamanaka, and E Suzuki, "Control on the formation of Si nanodots fabricated by thermal annealing/oxidation of hydrogenated amorphous silicon, Journal of Applied Physics, Volume 96, Pages 7532, 2004.

40. P. Photopolus and A Nassiopolulou, " Room and low temperature voltage tunable electroluminescence from a single layer quantum dots in betweentwo thin $\mathrm{SiO}_{2}$ layers", Applied Physics Letters” Volume 77, Pages 1816, 2000.

41. T. Kim, N. Park, K. Kim, G Sung, Y. Ok, T. Seong and C. Choi, " Quantum confinement effect of silicon nanocrystals in situ grown in silicon nitride films" Applied Physics Letters”, Volume 85, Pages 5355, 2004.

42. S. Thompson, C. Perrey, C. Carter, T. Beelich, J. Kakalios and U Kortshagen, "Experimental investigations in to the formation of nanoparticles in a nc-SI:H thin films" Journal of Applied Physics" Volume 85, Pages 5355, 2004.

43. K. Cho, N. Park, T. Kim, K. Kim, G Sung, "High efficiency visible electroluminescence from silicon nano crystals embedded in silicon nitride using a transparent doping layer “, Applied Physics Letters, Volume 97, Pages 034310, 2005.

44. R. D. Tilley, J. H. Warner, K. Yamamoto, I. Matsui and H. Fujimori, “ Microemulsion synthesis of monodisperse surface stabilized silicon nanocrystals". Chem Comm Volume 15, Pages 1833, 2005.

45. J. H. Warner, A. Hoshino, K. Yamamoto, R. D. Tilley, "Water-soluble photoluminescent silicon quantum dots." Chem. International Edition, Volume 44, Pages 4550, 2005.

46. J.P Wilcoxon and G.A Samara and P.N. Provencio, "Optical and electronic properties of Silicon nanoclusters synthesized in inverse micelles", Phys. Rev. B, Volume 60(4), Pages 2704, 1999.

47. J.P Wilcoxon and G.A Samara "Tailorable, visible light emission from silicon nano crystals”. Applied Physics Letters” Volume 74(21), Pages 3164, 1999.

48. J. Zou, R.K Baldwin, K.A Pettigrew and S.M. KAuzlarich, "Solution synthesis of ultrastable luminescent siloxane-coated silicon nanoparticle". Nano Letters, Volume 4(7), Pages 1181, 2004. 
49. R.K Baldwin, K.A Pettigrew, M.P. Augustine and S.M KAuzlarich. "Solution reduction synthesis of surface stabilized silicon nano particles". Chem Comm, Pages 1822, 2002.

50. V.A Schweigert and I. V Schweigert. "Coagulation in low-temperature plasmas". J Physics. D: Applied Physics, Volume 29, Pages 655, 1996.

51. U. Kortshagen and U. Bhandarkar, "Modeling of particulate coagulation in low pressure plasmas". Phys Rev E, Volume 60(1) Pages 887, 1999.

52. C. Cui and J. Goree, "Fluctuations of the charge on a dust grain in a plasma". IEEE Transactions on Plasma Science. Volume 22(2), Pages 151, 1994.

53. W. F Bergerson, J. A Mulder, R. P. Hsung and X-Y Zhu, "Assemble of organic molecules on silicon surfaces via the Si-N linkage." J. Am. Chem Society, Volume 121, Pages 454, 1999.

54. X-Y Zhu, J. A Mulder, W. F Bergerson, "Chemical vapor deposition of organic monolayers on Si(100) via Si-N Linkages" Langmuir Volume 15, Pages 8147, 1999.

55. X-Y Zhu, V. Boiadhiev, J. A Mulder, R. P. Hsung and R. C Major, "Molecular assemblies on Silicon Surface via Si-O Linkages." Langmuir, Volume 16, Pages 6766, 2000.

56. J. M Buriak, "Organometallic Chemistry on silicon and germanium surfaces" Chemical Reviews Volume 102(5) Pages 1271, 2002.

57. J. M Buriak, M. P. Stewart, T. W. Geders, M. J Allen, H. C Choi, J. Smith, D. Raftery and L. T. Canham, "Lewis acid mediated hydrosilation on porous silicon surface". Journal of Am. Chem. Society. Volume 121, Pages 11491, 1999.

58. L.C.P.M de Smwt, H. Zuilhof, E.J. R Sudholter, L. H Lie, A. AHoulton and B. R. Horrocks, Mechanism of the hydrosilation reaction of alkenes at porous silicon: experimental and computational deuterium labeling studies. Journal of Phys. Chem. B, Volume 109, Pages 12020, 2005.

59. R. K Baldwin, K.A Pettigrew, E. Ratai, M. P Augustine and S. M. Kauzalrich, "Solution reduction synthesis of surface stabilized silicon nanoparticles". Chem. Comm. Pages 1822, 2002.

60. R. D Tilley, J. H. Warner, K. Yamamoto, I. Matsui and H. Fujimori, "Microemulsion synthesis of monodisperse surface stabilized silicon nanocrystals". Chem. Comm. Volume 14, Pages 1833, 2005.

61. J. H. Warner, A. Hoshino, K. Yamamoto and R. D Tilley, "Water-soluble photoluminescent silicon quantum dots". Angew. Chem. Int. Ed. Volume 44, Pages 4550, 2005.

62. X. Li, Y He, S. S. Talukdar and M. T. Swihart, "Process for preparing microscopic quantities of brightly photoluminescent silicon nanoparticles with emission spanning the visible spectrum”. Langmuir Volume 19, Pages 8490, 2003.

63. F. Hua, M. T Swihart and E. Ruckenstein, "Efficient surface grafting of luminescent silicon quantum dots by photoinitiated hydrosilation". Langmuir Volume 21(3). Pages 6054, 2005. 
64. G. Natta, G. Mazzani and P. Corradini Alti Acad Naz Lincei Rend Sci. Fis. Mat. Nat. 25, 2, 1958.

65. H. Shirakawa, E. J. Louis, A. G. McDiarmid, C. K. Chiang and A. J. Heeger, Journal Chem. Soc. Chem. Comm. 577, 1997

66. J. Tsukamoto, A. Takahashi and Kawasaki, Japan. Journal of Applied Physics, 29, 125,1990

67. Maksudul M. Alam and Samson A. Jenekhe, Journal of Phy. Chem. B Volume 105(13), page 2479, 2001.

68. P.Bauerle, U.Mitschke, G. Gruner and G. Rimmel, Pure Applied Chem. Volume 71(11), page 2153, 1999.

69. I. B. Berlman, Handbook of Fluorescence Spectra of Aromatic Molecules, Second ed. (Academic Press New York, 1971).

70. J. H. Burroughes, D.D.C Bradley, A.R. Brown, R.N. Marks, K. Mackay, R.H. Friend, P.L Burn and A.B. Holmes. Nautre 347, 539, 1990.

71. W. Reppe Acetylene Chemistry U.S.Department of Commerce 188852-S 1959.

72. G. M. Kline Modern Plastics 24, 159, 1946

73. J.M Pearson and M. Stolka, Poly(N-vinylcarbazole), New York, Gordon and Breach Science Publishers, 1981

74. E.H Cornish, Plastics, 281963.

75. H. Block, M.A Cowd and S.W Walker, Polymer, 18, 781, 1977

76. S. Kirchmeyer and K. Reuter, Journal of Material Chemistry, Volume 14 page 2077, 2005.

77. J.Y. Kim, J.H. Jung, D.E. Lee et al, Synthetic Metals. Volume 126 page 311, 2002.

78. S.K.M Jonsson, J. Birgerson, X. Crispin, et al. Synthetic Metals. Volume 139, page 1, 2003.

79. J.Y Yang, D.H.K., J.L. Hendrics, M. Leach, R. Northey, D.C. Martin. Surfactant Templated poly(3,4-ethylenedioxythiophene) (PEDOT) Conducting Polymer on Micro fabricated Neural Probes. Acta Biomaterilia Volume 1, pages 125-136, 2005.

80. T. Aernouts, P.V., W Geens, J. Poortmans, P. Heremans, S. Borghs, R. Mertens, R. Andriessen, L. Leenders. Printable anodes for flexible organic solar modules. Thin solid films. Volume 451, pages 22-25, 2004.

81. V.G. Khomenko, V.Z.B., A.S. Katashinkii, Electrochimca. Acta Biomaterilia. Volume 50, pages 50-62, 2005.

82. Roman L. S, Mammo W, Pettersson LAA, Andersson MR, Ingana“s O. Adv. Material. Volume 10(10) pages 774-777 1998.

83. Granstrom M, Petritsch K, Arias AC, Lux A, Andersson MR, Friend RH. Nature. Volume 395 pages 257-260 1998.

84. Groenendaal LB, Jonas F, Freitag D, Pielartzik H, Reynolds JR. Adv Material. Volume 12(7), pages 481-94, 2000.

85. Scott JC, Carter SA, Karg S, Angelopoulos M. Synth Met. Volume 85(1-3), Pages 197-200, 1997. 
86. Kim J. S, Lagel B, Moons E, Johansson N, Baikie ID, Salaneck WR, Friend RH, Cacialli F. Synth Met. Volume111 pages 311-314, 2000.

87. Kim J. S, M. Granstrom, R. H. Friend, N. Johansson W. R. Salaneck, R. Daik, W. J Feast and F. Cacialli, Journal of Applied Physics. Volume 84, page 68591998.

88. Semiconductor Physics and Devices, Third Edition, Donald A. Nieman, The McGraw Hill Company.

89. Dan Yu, Gagan Aggarwal, et al. "Light Emitting Devices using Silicon Quantum Dots in PMMA". Organic Microelectronics and Optoelectronics Workshop" V-2009. 